Chapter 9

\title{
Describing Prostate Cancer Dynamics: Second Look at PSA-Doubling Time and PSA-Specific Growth Rate
}

\author{
Glenn Tisman \\ Additional information is available at the end of the chapter \\ http://dx.doi.org/10.5772/53179
}

\section{Introduction}

Physicians responsible for patient care focus on readily available clinical and trending laboratory data to help direct the patient's clinical course and evaluate efficacy of therapy. Most clinicians fail to incorporate newer parameters of tumor response such as tumor growth rate when evaluating patient treatment response. Available now, is a wealth of dynamic growth parameters that shed new light on tumor biology and should be used in clinical decisionmaking.

What follows is in part a review of former paradigms of prostate tumor growth. Later, focus is directed to newer techniques to assist in evaluating targeted drug effects on the kinetics of prostate and other cancers. The discussion introduces the concept of tumor or marker specific growth rate (SGR) and challenges historical results obtained by use of the classic tumor or marker doubling time (PSA-DT).

As we proceed with this discussion, a mobile device App for hand-held computers including the iPhone, iPad, or iPod is presented. This conveniently facilitates a more sophisticated tumor and marker analysis at the bedside or in the clinic.

\section{Historical perspective of tumor growth kinetics, exponential and Gompertzian kinetics}

Though there is occasional homage paid to Gompertzian tumor growth, for practical purposes, when we care for patients, tumors are frequently undergoing exponential expansion. 
In the absence of tumor mutation or perturbation by therapy the growth rate of exponentially growing tumors is constant. Rarely, there may be periods of interrupted growth.

Gompertzian growth [1, 2, 3] is best described by a sigmoid-shaped curve. At tumor initiation growth is occult, slow and remains subclinical for several years. A second phase is the rapid, clinically apparent exponential phase lasting for a few years followed by the slower terminal growth phase as the tumor approaches 35-40 doublings representing a volume approaching $1000 \mathrm{cc}$ or a tumor diameter of $10 \mathrm{~cm}$ Figure 1. The duration of tumor growth from inception is several years and for three quarters of that period the tumor is clinically undetectable. At the time of discovery, the oncologist is attending to the last quarter of tumor growth.

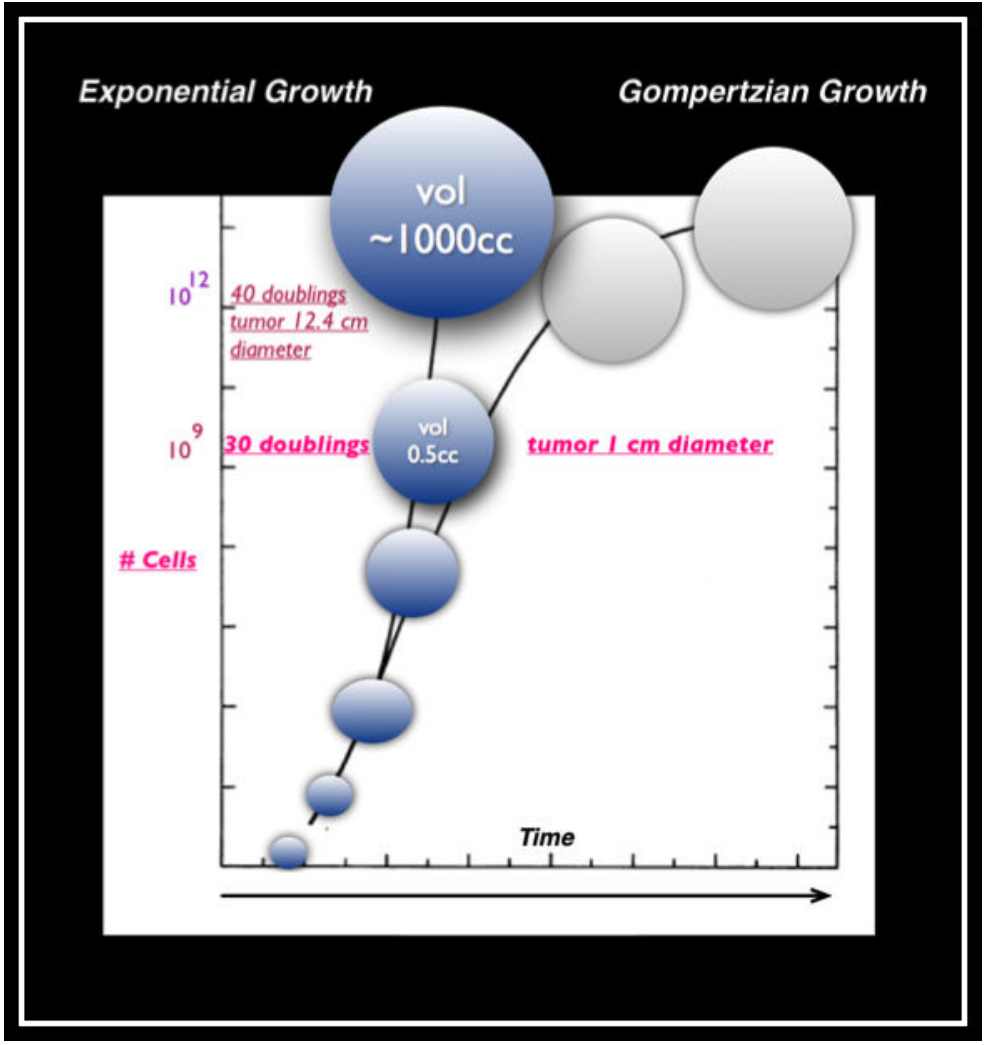

Figure 1. Note the differences between the exponential and Gompertzian growth curves. The lethal burden of tumor is approximately $1000 \mathrm{cc}$ or $\sim 35-40$ doublings. In the clinic when tumors reach $0.5-1 \mathrm{~cm}$ in diameter (30 doublings or $10^{9}$ cells) they are measurable and follow the exponential growth curve, the steeper the slope the larger the tumor specific growth rate (SGR). Nonetheless, many feel that when looking at the entire lifespan of malignant tumors (over several years) tumor growth may better be described by Gompertzian kinetics [3]. 


\section{Exponential growth}

In 1934 Mottram [4, 5] reported work on the rat tar wart. Tar warts are tar-carcinogen induced neoplasms of the skin starting 75-100 days after the continuous painting of the rat's neck with tar. Histologically, some warts appear benign while others are clearly malignant.

Using the tar wart tumor growth model, Mottram was the first to describe tumor expansion as exponential. Exponentially growing tumors graphically produce straight lines by plotting linear time on the $\mathrm{x}$-axis versus the log (at any base) of either tumor area, tumor cell number, tumor volume or tumor diameter on the y-axis see Figure 2.

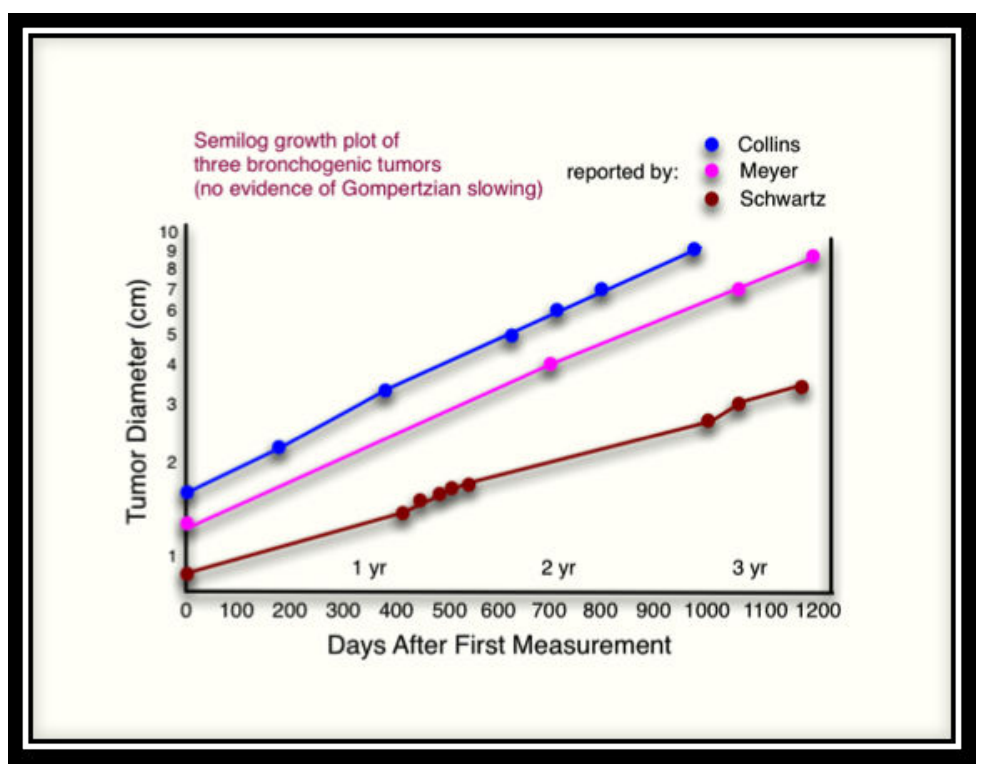

Figure 2. Friberg, Collins, Spratt, Steel, Schwartz affirmed that in the clinic, an exponential growth pattern adequately described tumor growth for most patients. A semi-log plot of tumor diameter vs. time illustrates the linear relationship characteristic of exponential growth.

Twenty years later Laird $[6,7]$ reported on the growth of transplanted tumors in the rat. Under her specific laboratory conditions, most tumor growth could be described in terms of the Gompertzian model. Her experiments lead her to accept that for her laboratory model; most transplantable, rapidly growing tumors could be described in Gompertzian terms.

Studies of tumor growth in clinic patients have been described in terms of both exponential and Gompertzian models. Nevertheless, several investigators reported data that was inconsistent with the Gompertzian model for the majority of their patients. These authors engaged routine imaging of both metastatic and primary pulmonary lesions in an attempt to resolve whether exponential growth could be confirmed in the clinic. Friberg, Collins, Spratt, Steel, Schwartz $[8,9,10,11,12,13]$ affirmed that in the clinic, an exponential growth pattern adequately described tumor growth for most patients Figures 2, 3. 


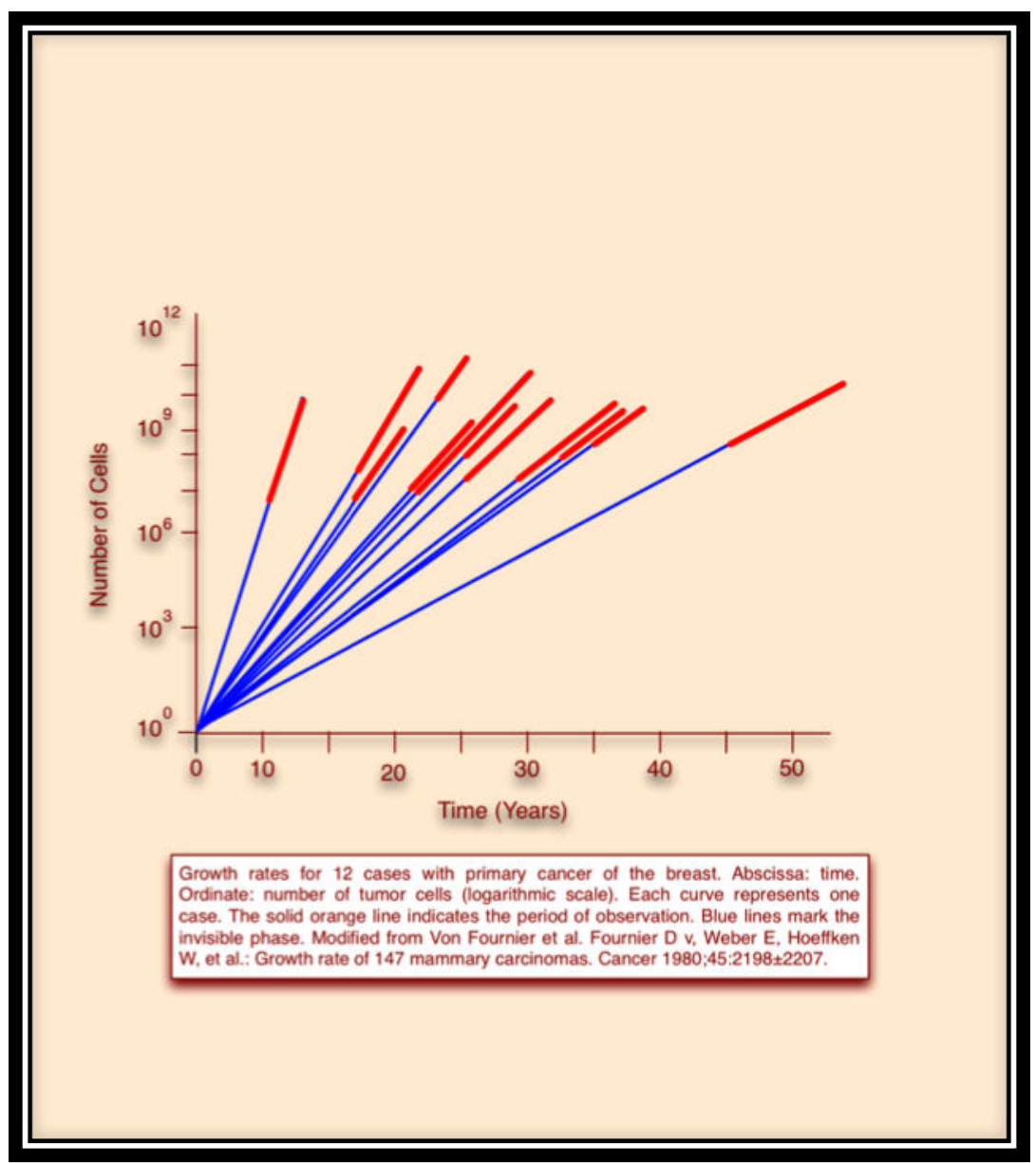

Figure 3. Von Fournier et al. confirm a straight-line (by semi-log plot) relationship for patients with breast tumors supporting the model of exponential growth model.

\section{The tumor marker as a surrogate for tumor growth exemplified by PSA and prostate cancer}

\subsection{PSA Velocity (PSA-V)}

PSA-V is the rate of change in serum PSA over time. PSA-V $=1 / 2\left(\left(\mathrm{PSA}_{2}-\mathrm{PSA}_{1} / \mathrm{t}_{1}\right.\right.$ in years $)+$ $\left(\mathrm{PSA}_{3}-\mathrm{PSA}_{2} / \mathrm{t}_{2}\right.$ in years)), where $\mathrm{PSA}_{1}$ is the first, $\mathrm{PSA}_{2}$ the second and $\mathrm{PSA}_{3}$ the third PSA measurement. Time represents the interval (in years) between PSA measurements. It is recommended that three PSA measurements obtained over 24 months yields optimal accuracy. A PSA-V exceeding $0.75 \mathrm{ng} / \mathrm{ml} /$ year is highly predictive of prostate cancer. PSA-V is more 
useful than PSA doubling time (PSA-DT) in the pretreatment setting to help identify those men with life-threatening disease [14].

Studies confirm that the PSA tumor marker reflects prostate tumor growth and PSA dynamic changes are useful for predicting clinical outcome in several situations such as tumor recurrence and overall survival [15].

Klotz [16] reviewed the value of PSA as a tumor marker in patients with prostate cancer. He noted that use of a single serum value of PSA is inadequate for predicting patient survival. However, the PSA-V as ng/ml/yr. was a marker of disease biology. D'Amico [17] included preoperative PSA-V in determining subsequent risk of death from prostate cancer in 1095 men with clinically localized prostate cancer that underwent prostatectomy and radiation therapy [18]. A PSA-V $>2 \mathrm{ng} / \mathrm{ml} / \mathrm{yr}$ the year before prostatectomy, was associated with lymph node metastases, an advanced pathologic stage, and high-grade disease. This threshold level of PSA-V was associated with a significantly shortened time to recurrence, death from prostate cancer, and death from any cause. Strikingly, men with a PSA rise of $>2.0$ $\mathrm{ng} / \mathrm{ml}$ had prostate cancer-specific mortality rates nine times those with a PSA-V $<2 \mathrm{ng} / \mathrm{ml}$.

\subsection{Tumor marker Doubling Time (DT)}

Miyamoto [19] studied the growth of hepatic metastases in colorectal cancer patients. He established that a tumor marker could accurately reflect tumor volume and its changes. Using the CEA tumor marker he reported an almost equal and parallel correlation between CEA doubling time and hepatic tumor volume doubling time.

PSA-DT Figure 4 is the time it takes for the serum PSA to double. Evidence indicates PSADT closely mirrors prostate tumor volume doubling time. Kato et al. in 2008 [20] undertook an attempt to correlate prostate tumor volume to serum PSA level. Kato's group calculated that for each $\mathrm{ng} / \mathrm{ml}$ increment of serum PSA, there was a $0.302 \mathrm{cc}$ increase in total tumor volume and a $0.7 \%$ increase in relative tumor volume. Total tumor volume in cc was given as $\mathrm{V}(\mathrm{cc})=3.476+0.302 \times$ PSA $(\mathrm{ng} / \mathrm{ml})$ while the percent tumor volume Volume $(\%)=11.331+$ $0.704 \times$ PSA (ng/ml).

Babaian et. al. [21] reported that multivariate regression analysis of tumor volume as a function of PSA, grade and stage demonstrated that log PSA had the strongest association with tumor volume. Tanaka [22] reported that among significant preoperative and postoperative parameters, calculated cancer volume remained an independent predictive parameter in multivariate analysis $(\mathrm{P}<0.01)$. Tumor volume, as calculated by preoperative parameters, was an independent predictor of biochemical recurrence in patients who had undergone radical prostatectomy. Vollmer et al. [23] used a compartmental model and first order kinetics to develop the calculation necessary to relate serum PSA to tumor volume. They found that the resulting model was a good fit to the observed kinetic data of PSA measured after biopsy or prostatectomy. The model also predicted a linear relationship between PSA and the sum of volumes of benign and malignant tissues.

Until evidence to the contrary, it is assumed that similar to colorectal tumors and CEA, there is a reasonable relationship between serum PSA and its kinetics allowing its use as a predictor of changes of prostate tumor volume and growth kinetics. 
An important point when using serum PSA in calculations is that an exact interval for testing remains controversial, some investigators stress that the interval between PSA-DT determinations should approach 3-6 months [24] to limit error due to random variation of PSA values. Using a third generation highly sensitive PSA assay, our laboratory changes in PSA are precise to the third decimal point and allow educated decision-making based on monthly determinations.

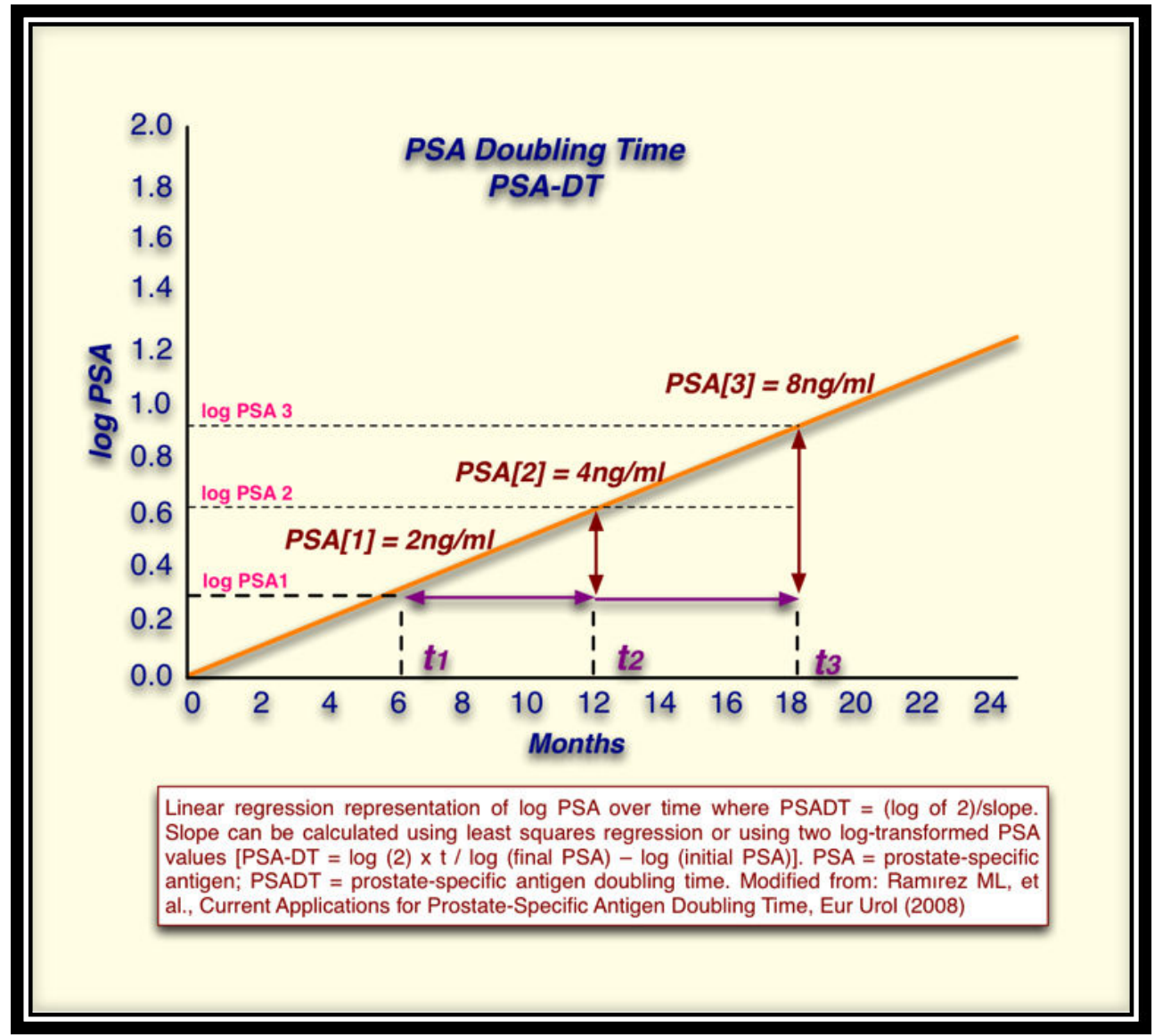

Figure 4. This figure is a semi-log plot of logs PSA (y-axis) vs. time (x-axis) [26]. Note the linear relationship, indicating that the rise of PSA values follows an exponential expansion of PSA.

Historically, PSA kinetics for watchful waiters included PSA-DT. A PSA-DT of $>10$ yr. can be considered favorable; a PSA-DT of <3-4 yr. suggests a change in biology and consideration should be given to an alternative therapy [25]. PSA kinetics should always be combined 
with other diagnostics such as endorectal ultrasound; endorectal MRI, digital rectal exam and repeat prostate biopsies approximately every 6-12 months.

\subsection{PSA-DT as a surrogate for drug activity}

PSA is one of the major androgen receptor-dependent target genes [27], and clinical monitoring is used to detect early stage disease as well as the emergence of recurrent tumor after therapy [28, 29, 30] and changes mirror changes in tumor bulk and indicate response to drugs. The graphic representation of PSA-DT is illustrated and its formula is given in Figure 4 [26].

Kelloff et al. [31], reviewed the use of PSA-DT as a surrogate for tumor response to drugs in patients with prostate cancer. They concluded that protocols that demonstrate significant changes in PSA-DT might be used to support accelerated approval of newer therapies. There is data to suggest PSA-DT in castrate resistant patients is predictive of outcome after chemotherapy [32]. An important caveat is expressed by Newling's review [33] of the subject which concluded that though dynamic changes in the PSA such as PSA-DT are commonly used in clinical trials of new drug therapies, PSA-DT might be affected by other factors including assay variations and false elevations of serum PSA caused by irritation of bladder catheters, prostatitis and cystitis. A substantial incidence of transient elevations of PSA (55\%) was reported following combined external beam radiation and brachytherapy for prostate cancer [34]. These complicating issues should always be considered before PSA-DT is used to modify therapy.

Most recently, newer targeted and immunotherapies were found to produce paradoxical effects on PSA kinetics. Newling [33] argues that PSA should therefore be used as a secondary end point while overall survival still remains the gold standard in evaluating therapeutic efficacy for patients with hormone refractory disease.

\section{Defining PSA response}

Investigators participating in new prostate cancer drug trials commonly define PSA response according to the Bubley guidelines [35] for phase II clinical trials in androgen-independent prostate cancer. The guidelines qualify the following categories of PSA: PSA normalization, PSA $<=0.2 \mathrm{ng} / \mathrm{ml}$; PSA decrease, PSA decline $\geq 50 \%$, confirmed by a second PSA value 4 or more weeks later; PSA progression, PSA $\geq 25 \%$ increase over the baseline (and an increase in the absolute value PSA level by at least $5 \mathrm{ng} / \mathrm{mL}$ ). Though useful for evaluating clinical trials, these PSA changes lack sensitivity when evaluating subtle drug effects vs. prostate tumor growth $[36,37,38]$.

Therasse [39, 40], in his thesis reports on MRI and PSA as tools in a RECIST evaluation used to define tumor response in prostate cancer patients with measurable soft tissue lesions. When comparing MRI soft tissue responses to serum PSA changes, the correlation of PSA and MRI showed agreement in 14 of the $20(70 \%)$ patients. 


\section{PSA-DT and Survival of prostate cancer patients}

The importance of PSA-DT in predicting survival is illustrated by Freedland et al. [41] Figure 5. This chart presents data for a group of patients experiencing biochemical recurrence of PSA after prostatectomy. Under these circumstances, PSA-DT clearly defined prostate cancer survival into four groups: 1) PSA-DT >=15 months, 2) PSA-DT 9-14 months 3) PSADT 3-8.9 months, 4) PSA-DT $<3$ months. For this study, PSA-DT is clearly a surrogate for prostate cancer-specific survival.

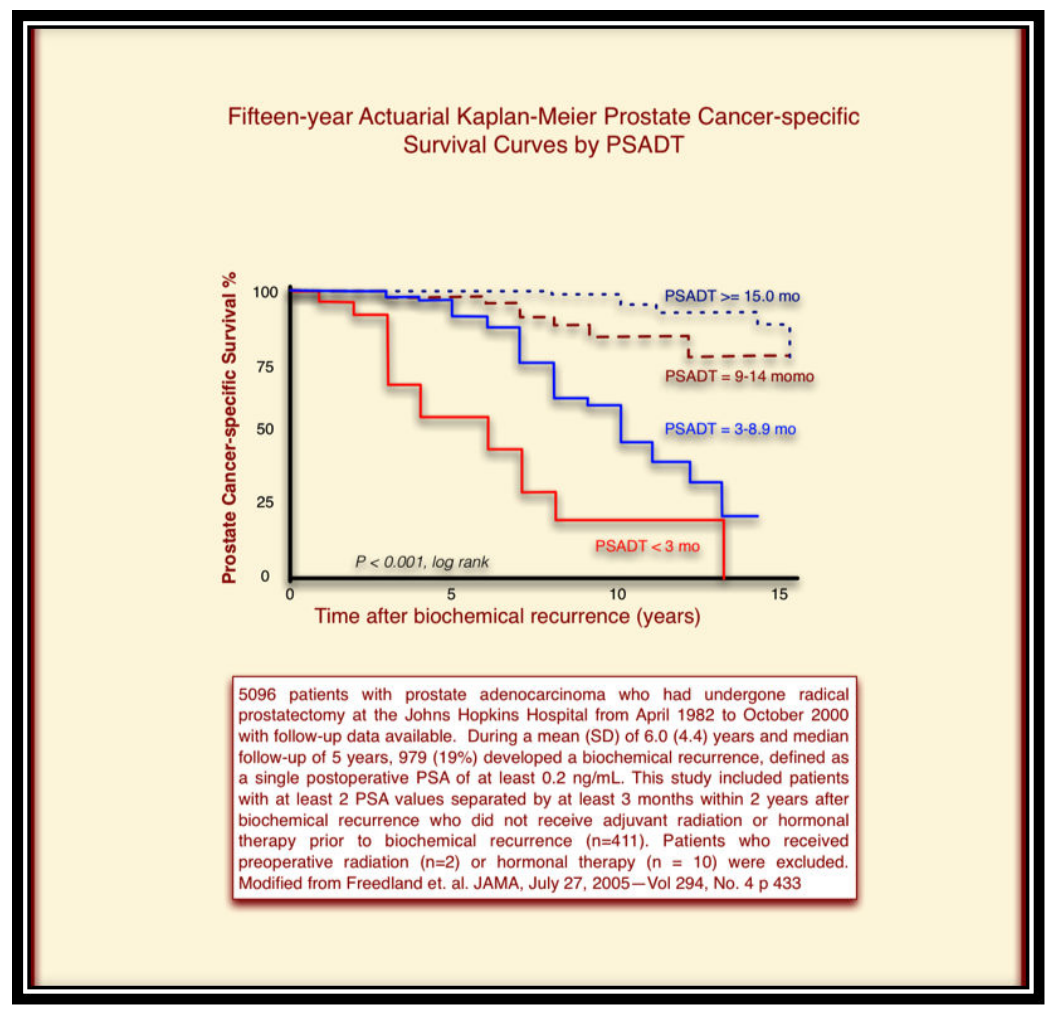

Figure 5 .

\section{PSA in the era of biologic and targeted therapy}

A wealth of data establishes PSA as a marker of tumor aggressiveness, tumor stage, drug response and survival. Controversy and concern persists regarding PSA's role as a marker of disease stabilization and response induced by cytostatic and immunotherapies when 
compared to cytolytic therapies. An evaluation of the difficulties surrounding PSA interpretation has been addressed [42].

Two vaccine trials, Sipuleucel-T (Provenge) [43, 44, 45] and the TRICOM PROSTVAC [46, 47] demonstrated a significant overall survival benefit without any consistent decline in PSA, raising questions about the value of PSA response for non-hormonal, non-cytotoxic therapies. In addition, wide fluctuations have been observed in PSA values due to a transient effect of some drugs on PSA production seemingly independent of cell proliferation. The independent, non-proliferative effect of drugs on PSA expression should be considered when interpreting PSA response data. These aberrant PSA effects must be considered together with imaging results and clinical evaluation of the patient. Nevertheless, it has been consistent that post therapy a $>50 \%$ PSA decline in pre-treatment PSA carries a significant overall survival advantage $[48,35]$.

Kelly [48] reported on 110 assessable patients treated on seven sequential protocols at Memorial Sloan-Kettering Cancer Center for hormone-refractory prostate cancer a statistically significant survival advantage in 110 patients with $>50 \%$ PSA decline ( $>25$ months survival) versus those without a 50\% PSA decline ( 8.6 months survival). These results suggest that post therapy PSA declines can be used as a surrogate end point to evaluate new agents in hormone-refractory prostate cancer and criteria for response need prospective validation for phase III trials. Smith et al. [49] showed that a PSA decline $>50 \%$ for at least 8 weeks resulted in a longer mean survival time of 91 weeks versus 38 weeks for patients showing a smaller PSA reduction. An improved PSA response was associated with prolonged survival in the TAX 327 study (Docetaxel plus Prednisone or Mitoxantrone plus Prednisone for Advanced Prostate Cancer), with a median survival of 33 months when the PSA was normalized $(<4 \mathrm{ng} / \mathrm{mL})$ versus 15.8 months for an abnormal PSA [50, 51].

Heidenreich [52], the chair of the European Association of Urology oversaw the EAU 2012 Prostate Cancer Guidelines. He acknowledged that the PSA has been validated to be the most clinically useful tumor marker of treatment failure following local therapy and of tumor response as well as of tumor progression following hormonal treatment.

\section{Assessment of molecularly targeted, cytostatic or anti-angiogenic agents}

Bellmunt [53] and others expressed concern that PSA response criteria are not established to properly evaluate molecularly targeted cytostatic or anti-angiogenic agents [54]; therefore, certain drug-specific limitations may exist when using PSA or PSA-DT as an indicator of progression or response. One clear example was noted in a study of sorafenib (Nexavar) in castrate resistant prostate cancer, in which two patients with PSA progression were found to have dramatic resolution of bony disease [55]. Therapy-associated PSA "surge" has been described after effective chemotherapy. PSA surge occurs with Samarium ${ }^{153}$ radiotherapy, androgen deprivation and chemotherapy and is generally transient. The surge may be due to rapid lysis of prostate cancer cells thus spilling intracellular contents into the intravascular 
space [56]. Similarly, 10 of 16 patients who discontinued sorafenib and did not receive other therapy demonstrated post-discontinuation PSA declines of 7-52\% [57]. The review by Bellmunt $[58,59]$ notes that several targeted therapies caused prolongation of the PSA-DT as well as significant suppression of PSA levels. The era of targeted therapy for prostate cancer is just beginning and will require changes in how we interpret PSA kinetics.

\section{Considerations in evaluating tumor growth effects of targeted therapies}

Newer targeted therapies are often cytostatic or cytolentic (slowing proliferation) [60], resulting in disease stabilization, improved quality of life and extended survival. Examples of such drugs include sorefinib (Nexavar) [61], axitinib (Inlyta) for renal cell carcinoma [62], and mTOR inhibitors (everolimus (Afinitor) [63] and temsirolimus (Torisel)). Dasitinib (Sprycel) and sorefinib (Nexavar) are active in prostate cancer. Dasatinib is active in chronic granulocytic leukemia and GIST, inhibits BCR/ABL tyrosine kinase, KIT, PDGFR and Src tyrosine kinase amongst other targets. The Src tyrosine kinase is instrumental in driving hormone-independent prostate cancers [64]. Dasatinib is active in castrate resistant prostate cancer and may be administered safely with docetaxel $[65,66]$.

These newer therapies target not only the tumor cell but also modify the supporting stroma and microvasculature. The cytostatic/cytolentic effects may leave the tumor dimensionally intact, stable on imaging studies but with slower or absent growth for extended periods of time. Some imaging techniques such as PET and MRI [67], able to quantify such metabolic effects, may enhance clinical evaluation while CT images appear unchanged.

There is mounting evidence that stabilization of tumor growth significantly prolongs overall survival to a degree similar to patients experiencing an objective response judged by RECIST or RECIST 1.1 criteria (Response Evaluation Criteria in Solid Tumors). This raises concern and new calls for modification of current RECIST categories to include new definitions for targeted responses [68].

Simple reductions in PSA levels as defined by Bubley [35] have not yet been validated as a surrogate end point for use in clinical trials of agents with novel mechanisms of action. As indicated, cytotoxic chemotherapy alone, in combination with molecular-targeted agents, or the sole use of targeted therapies, produces different and at times transient and paradoxical changes in serum PSA and further studies are needed to further define this issue.

As questions have emerged concerning the utility of PSA levels as a surrogate end point, the Prostate Cancer Clinical Trials Working Group reviewed the criteria for outcome measures in clinical trials that evaluate systemic treatment for patients with progressive prostate cancer. Recommendations conclude that PSA responses may be delayed in trials of non-cytotoxic agents, and rising PSA levels in the absence of other signs of progression should not lead to discontinuation of trials. This recommendation might lead to much consternation between the patient and doctor where discussion of the latest PSA value is often the primary subject during follow-up visits. 


\section{Projected tumor size and projected PSA uncover hidden drug activity}

Now that surrogacy of static values of PSA and PSA-DT is being questioned for targeted therapies, new techniques of response evaluation are under study. One attempt to quantitate treatment efficacy redirects attention from PSA-DT to PSA-specific growth rate (PSASGR) [69, 70, 71]. Generally ignored, projected tumor and marker value play a particularly important role in uncovering and quantifying hidden, cytostatic or cytolentic drug effects. Projected tumor volume or marker value is calculated prior to the initiation of therapy and based on the specific growth rate constant (SGR) before the start of therapy. The projected value is illustrated in Figure 7. This growth projection captures the inherent tumor SGR before therapy and predicts what the outcome (projected tumor or marker volume/value) would be at any future date in the absence of treatment or tumor mutation. Older cytotoxic drugs, when effective, inhibit innate growth by programmed cell death and apoptosis resulting in autophagy and tumor cell lysis $[60,72]$. This results in a measurable reduction of tumor size. Interestingly, these drugs are often in part cytostatic or cytolentic and depending on dose may result in stable disease. Keep in mind that prolongation of cytostatic or cytolentic suppression by any drug may eventually induce cytotoxicity and cell lysis [60] Figure 6.

Different combinations of static/lytic drug activity may result in reduced tumor/marker size or complete tumor growth inhibition without clinically detectable change in tumor size. Under these circumstances, use of projected growth uncovers hidden suppression of proliferation. A common clinical scenario occurs when during treatment, a tumor increases in size but much less than projected. Unless the clinician calculates what the projected tumor size should be, the true degree of tumor suppression is not appreciated Figure 7.

\subsection{Mathematical relationships of exponentially growing tumors and projected tumor marker or tumor size/volume}

The mathematical expression for exponential expansion of growth is: $V_{t}=V_{0} e^{\alpha t}$ where the tumor volume at time $\mathrm{V}_{\mathrm{t}}$ is predictable and is the product of the starting tumor volume $\left[\mathrm{V}_{0}\right]$ and $[e=2.71828$, the base of the natural logarithm raised to the product of the specific growth rate constant $\alpha$ or (SGR) and the duration of growth $\Delta \mathrm{t}$ or $\left(\mathrm{t}_{1}-\mathrm{t}_{0}\right)$ ].

This is given as $\mathrm{V}_{\mathrm{t}}=\mathrm{V}_{0} \mathrm{e}^{\mathrm{SGR}{ }^{*} \Delta \mathrm{t}}$ and mathematical rearrangement yields $S G R=\frac{\ln \left(\frac{V 2}{V 1}\right)}{t 2-t 1}$

Inhibitory drug effects slow SGR and are precisely quantifiable by calculating changes of SGR and the tumor size before and after therapy. Tumor size after therapy should be compared to the projected tumor size the same time after therapy. The current standard for clinical oncologists is comparison of tumor size before and after therapy while neglecting comparison with the projected tumor size. Differences between post therapy tumor size and the post therapy projected tumor size are the clue to hidden responses that are almost never evaluated by the clinical oncologist. These often-subtle differences between projected and post therapy tumor sizes may reveal hidden growth stimulation (mutation or idiosyncratic drug effect) as well as subtle growth inhibition, which may lead to prolonged clinical stability. 
The following relationships, extracted from Mehrara's analysis [69,70,71] define projected tumor volume: $\int_{t i}^{t} \Delta S G R(t)^{*} d t=\ln \left(\frac{V n}{V i}\right)-\ln \left(\frac{V t}{V i}\right)$ where $\mathrm{V}_{\mathrm{n}}=$ projected tumor volume, $\mathrm{V}_{\mathrm{t}}=$ volume of tumor at the time of response evaluation and $\mathrm{V}_{\mathrm{i}}$ is the volume at the initiation of therapy. The tumor response or $T R=-\ln \left(V_{t} / V_{n}\right)$ where $V_{t}$ is the volume of treated tumor and $\mathrm{V}_{\mathrm{n}}$ is the hypothetical or projected tumor volume, both evaluated at the time of efficacy assessment. These relationships are the model for the growth kinetics of exponentially growing tumors and generally require the use of at least a handheld computer to facilitate evaluation in the clinic. This is further discussed in the appendix.

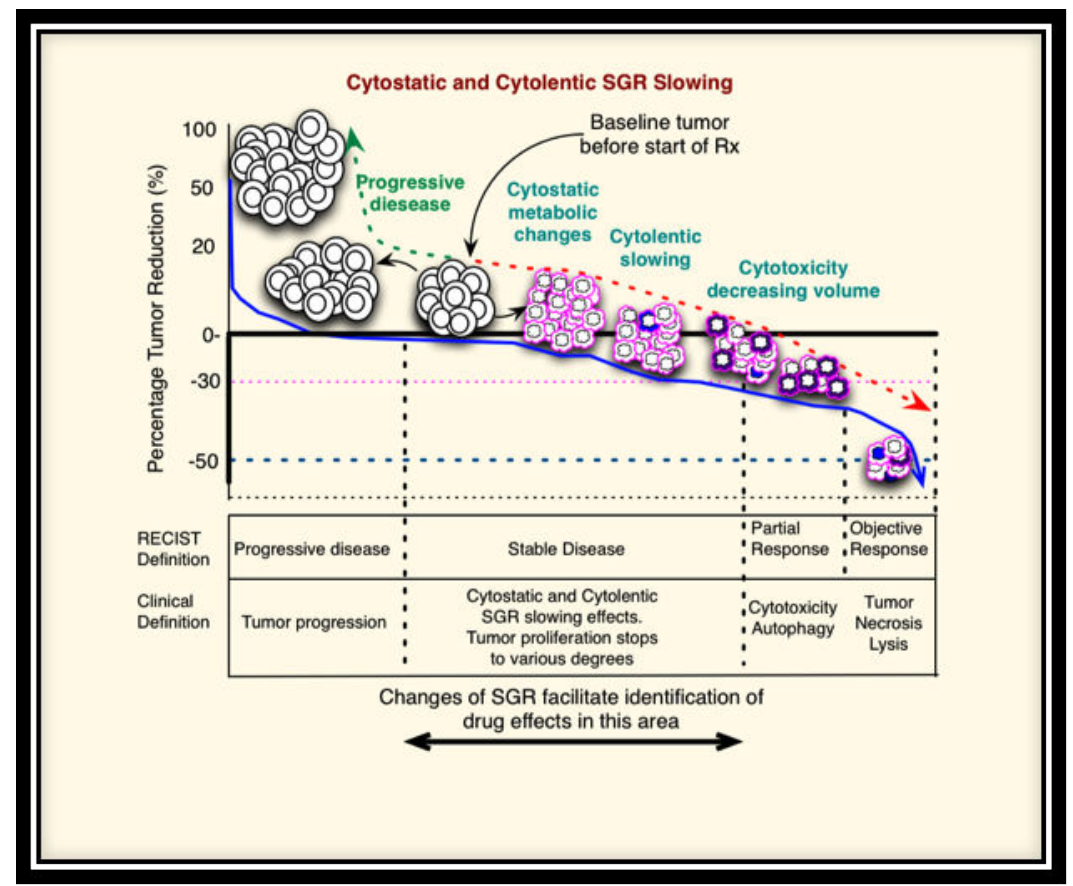

Figure 6. In vitro and in vivo, a clear distinction between cytostatic and cytolytic drugs does not exist. Low-dose cytolytic chemotherapy may exert cytostasis or so-called cytolentic slowing of cell proliferation leading to cell lysis, while targeted therapy's prolonged cytostatic metabolic effects (or large doses of targeted therapy) may induce cytolysis and autophagy (autophagocytosis). Regardless of mechanism of cell inhibition, the SGR and the TR (treatment response) calculations clearly and objectively define and quantitate drug efficacy (TR value).

Picture a $4.0 \mathrm{~cm}$ diameter $(14.1 \mathrm{cc})$ pulmonary metastasis. At the time of discovery two months before the start of therapy the tumor was $3 \mathrm{~cm}(33.5 \mathrm{cc})$. The pre-therapy SGR for this tumor $=1.46 \% / \mathrm{d}$ (tumor volume was expanding by $1.46 \% / \mathrm{d}$ ). Sixty-one days of therapy was administered and the tumor grew to $4.5 \mathrm{~cm}$ (47.7 cc). SGR decreased from $1.46 \% / \mathrm{d}$ to $1 \% / \mathrm{d}$. Clinicians unaware of SGR and the projected tumor volume at this point might declare drug resistance however; the projected tumor volume was actually $80.6 \mathrm{cc}$ and the tu- 
mor reached only $47.7 \mathrm{cc}$. Even though the tumor grew, therapy was significantly effective in slowing growth (59\% of intrinsic tumor growth was inhibited)! The parameter for treatment efficacy, TR was +0.5 . A positive value for TR means that therapy had some inhibitory activity against the tumor, the larger the value the better. A negative value means therapy was associated with growth stimulation. The value of TR is useful as an objective standard comparator to help evaluate efficacy between different treatments.

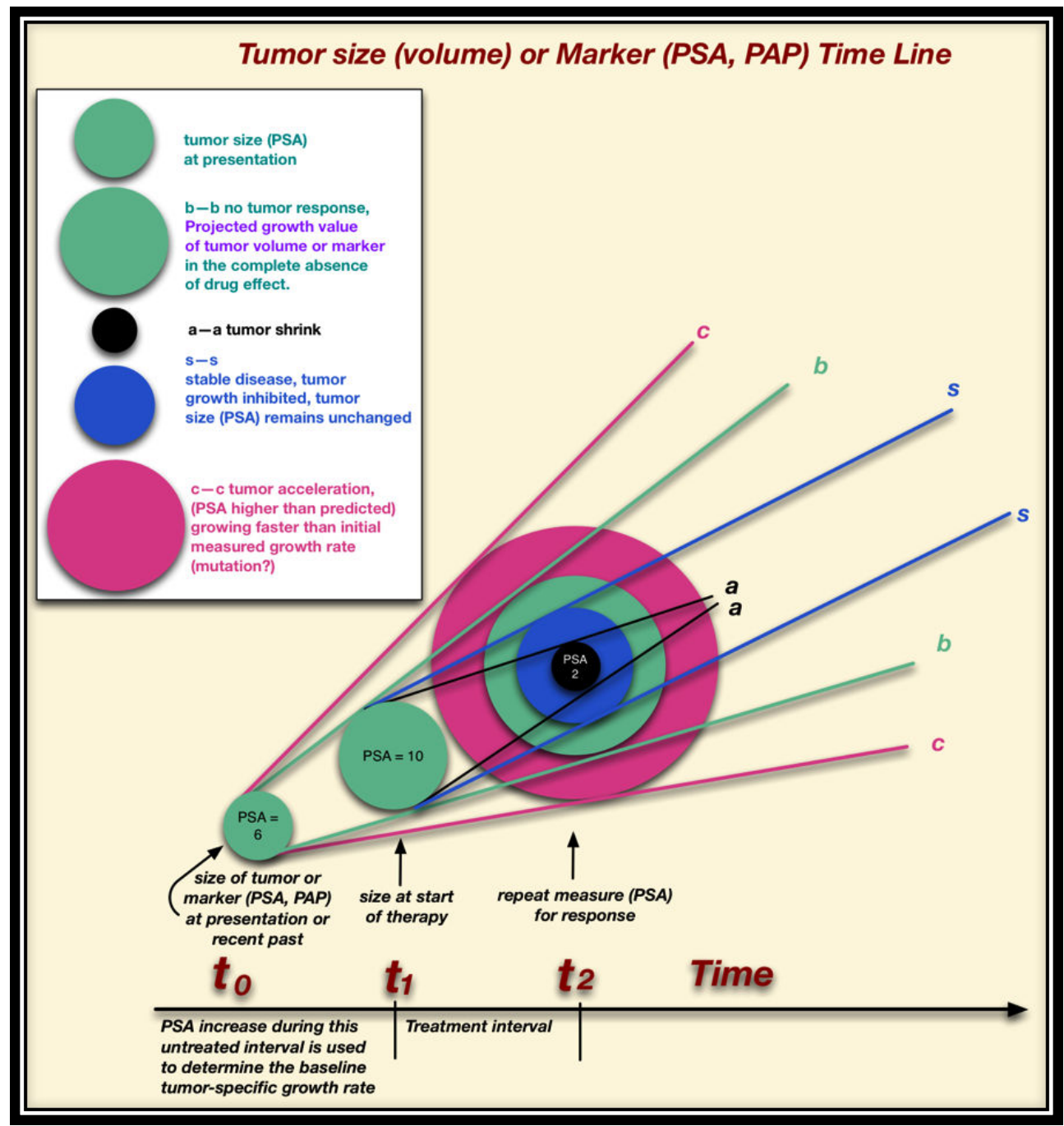

Figure 7. Tumor size (volume) or Marker (PSA, PAP) Time Line 
Figure 7 illustrates potential tumor responses to drug treatment. Some of the responses such as positive and negative deviation from the projected PSA value or projected tumor volume are routinely overlooked in the clinic because projected sizes for these parameters must be calculated in advance (projected volume is illustrated by the largest $b$ - $b$ green circle at $t_{2}$ ). SGR is calculated based on tumor or PSA growth between $t_{0}$ and $t_{1}$. Deviations from projected values reveal subtle drug-tumor interactions. In the appendix we discuss straightforward evaluation of all five-treatment outcome scenarios illustrated above by a hand-held computer.

Until now, most attempts to capture drug effects vs. prostate tumors employed changes of PSA-DT. However, Mehrara [70] presented newer assessments of PSA-DT compared to PSA-SGR that cast doubt on the validity of that historic collection of work.

What follows is a general listing of consequences of drug-tumor interaction. These potential tumor or marker responses Figures 6, 7 are important to understand because subtle changes in tumor proliferation may be the only drug-induced tumor response and may go unnoticed when evaluating targeted therapy by RECIST/RECIST 1.1 response criteria.

\subsection{Targeted therapies might require SGR calculations to evaluate the full spectrum of tumor response}

Figures 6, 7 display tumor responses evaluable in the clinic. RESIST 1.1 criteria follow for comparison.

1. Disease stabilization (complete inhibition of pre-therapy SGR)

The marker or tumor's inherent growth rate is inhibited causing it or its surrogate marker value to remain unchanged during therapy.

2. Uninterrupted growth

The tumor or marker continues its calculated pre-therapy growth rate without change during therapy. The growth noted in the surrogate marker or tumor after therapy is predictable and equal to the projected tumor growth based on the calculated SGR before the start of therapy.

3. Tumor "response" of varied degree (note: at the time of response evaluation the tumor may be larger than the pre-therapy value)

Tumor or marker growth is inhibited and at post-therapy response evaluation the tumor or its surrogate marker is less than the projected value. This response may be difficult to identify since the tumor or its marker may have reached a size greater than before the start of therapy however, tumor or marker post therapy is not as large as projected based on the pre-therapy SGR Figure 7. A computer calculation comparing pre- and post-therapy SGR is required to accurately quantify this response category. TR (treatment response) is easily calculable and offers an objective and continuous value for the degree of response. TR is used as either a "tumor response" or "tumor marker response", to quantitate the effect of thera- 
py. This continuous variable is useful to directly compare treatment efficacy between differing therapies.

Mehrara [71] defined some limitations for the current use of treatment response including: 1) PR and CR as defined in RECIST and other methods are no longer of value for quantifying responses to cytostatic/cytolentic drugs. Combinations of cytolytic and cytostatic/cytolentic therapies add further difficulty to response interpretation. A further problem arises when drugs are used at the extremes of dosing where tumor-killing activity may change from cytostatic/cytolentic to cytolytic and vise versa. 2) Classically, no consideration is given to the persistence of tumor SGR and or its inhibition during the course of therapy. Clinically, this is a trap for the oncologist if response is based solely in terms of whether the tumor marker or size is decreased at the end of therapy 3) The advantage of TR as a continuous variable (as opposed to a discrete variable used to compartmentalize responses such as CR, $\mathrm{PR}, \mathrm{SD}$ ) is that TR is a measurement of inhibitory (+TR) as well as accelerating (-TR) drug effects and is directly comparable between therapies and independent of mechanism of drug action.

A simple statement that the marker or tumor is larger post therapy is no longer adequate to evaluate tumor responses.

4. The size of the tumor or its surrogate marker decreases after therapy.

This may be a partial or complete return to normal, manifest by partial or complete disappearance of tumor/marker abnormality.

\section{Tumor acceleration and deceleration}

Tumor acceleration occurs when the tumor or marker growth rate (SGR) after therapy is greater than the pre-therapy or baseline SGR and SGR = (SGR after Rx - SGR before Rx) / $(\mathrm{t} 2-\mathrm{t} 1)$ is a negative value. Tumor growth rate acceleration is positive and may indicate the presence of a tumor-accelerating mutation or an unexpected untoward drug effect.

Tumor deceleration occurs when SGR before therapy is greater than SGR after therapy and is expressed as: $S G R$ deceleration $=(S G R$ after $R x-S G R$ before $R x) /(t 2-t 1)$ this is a negative value.

The rate of change calculations are based on the pre-therapy calculated SGR and its rate of change is calculated at the end of therapy and is expressed as: Acceleration or deceleration of the SGR: $\triangle S G R / \Delta t$. Or EDITOR use $\left(\mathrm{SGR}_{2}-\mathrm{SGR}_{1}\right) /\left(\mathrm{T}_{2}-\mathrm{T}_{1}\right)$.

Note: In the presence of multiple tumor targets the sum of tumor diameters or volumes is used as an approximation. Clonal heterogeneity (a mosaic of tumors growing at different growth rates and or demonstrating a mixed response) may make some tumors inadequate for analysis.

In 1999 an attempt to write a specific dogma evaluating tumor response resulted in the RECIST 1.0 criteria, later updated 2009 as RECIST 1.1 [73]. Note the absence of drug-response based on the concept of projected tumor growth.

RECIST 1.1 criteria 
Complete Response (CR): Disappearance of all target lesions. Any pathological lymph nodes (whether target or non-target) must have reduction in short axis to $<10 \mathrm{~mm}$.

Partial Response (PR): At least a 30\% decrease in the sum of diameters of target lesions, taking as reference the baseline sum of diameters.

Progressive Disease (PD): At least a 20\% increase in the sum of diameters of target lesions, taking as reference the smallest sum on study (this includes the baseline sum if that is the smallest on study). In addition to the relative increase of $20 \%$, the sum must also demonstrate an absolute increase of at least $5 \mathrm{~mm}$. (Note: the appearance of one or more new lesions is also considered progression).

Stable Disease (SD): Neither sufficient shrinkage to qualify for PR nor sufficient increase to qualify for PD, taking as reference the smallest sum diameters while on study.

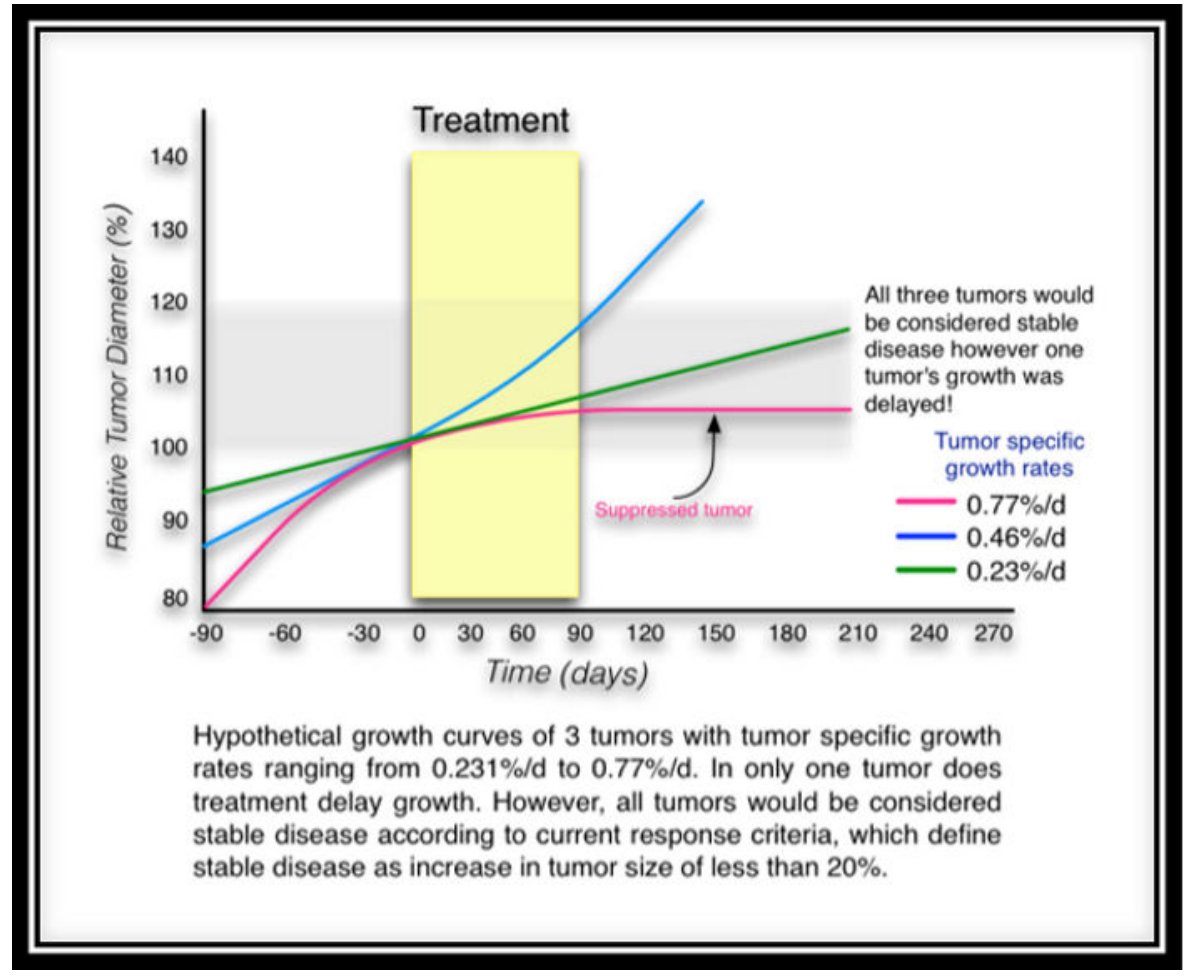

Figure 8. Weber [74] reveals the difficulty of classifying real growth inhibition within the RECIST1.1 criteria of stable disease. Real or suppressed tumor growth is illustrated by the pink growth curve only.

Weber noted that the RECIST 1.1 disease stabilization category does not differentiate between a drug that slows tumor growth and the complete lack of drug effect Figure 8 . The RECIST 1.1 definition for disease progression is $>5 \mathrm{~mm}$ absolute increase in size in addition 
to $>20 \%$ increase compared with the nadir. In this figure, though all three tumors do not meet the progressive disease criteria and thus would be termed stable, the growth of the third was slowed by therapy. Though all three are termed stable, note the subtle difference between the two tumors showing a continued and uninterrupted pre-therapy growth rate (SGR), compared to the slowed growth rate of the tumor depicted by the red line. Surely there is a drug effect vs. the red tumor. This active drug could be overlooked in spite of its potential to increase survival if maintained for a sufficient period of time.

In a review of a group of patients treated with targeted therapies, Tourneau [75] revealed clinical evidence where investigators overlooked subtle cytostatic/cytolentic (slowing of SGR) drug activity Figure 6, 8. The group analyzed 50 patient participants in 18 targeted therapy drug trials. Among the 44 patients who withdrew from study because of disease progression according to the investigators' assessment, 18 patients $(41 \%)$ demonstrated a favorable slowing trend in tumor specific growth rate. Among the 18, 5 had disease progression according to RECIST 1.1 according to retrospective reassessment of on-study imaging and occurrence of no new lesion during study treatment. Their preliminary evaluation concluded that a substantial proportion of patients treated with targeted agents were removed from protocol in spite of possibly benefitting from therapy.

Ferte et al. [76] studied metastatic renal cell carcinoma patients treated with sorafenib (Nexavar) and everolimus (Afinitor). Analysis of tumor SGR clearly revealed drug effects that would have been missed had RECIST response criteria been applied. Tumor response was assessed before, during, at the time of tumor progression and after drug discontinuation. Tumor growth rate was computed by dividing tumor shrinkage by the time between two related evaluations (\% RECIST x 100 /day).

In two different patient populations (IGR and TARGET) tumor growth rate significantly decreased following sorafenib (-23.6 vs. 20 (IGR) and -19 vs. 22 (TARGET)) and everolimus ( -5.2 vs. 30 (IGR)). The great majority of patients (IGR) had a decrease in the tumor growth rate during vs. before therapy, regardless of the RECIST evaluation, both with sorafenib $(28 / 29)$ or everolimus (36/37). Growth rate after sorafenib or everolimus interruption was significantly higher than at the time of progression in both settings (IGR) (14.6 vs. 31 and 17.9 vs. 32.1 respectively). No significant difference was observed between growth rate before or after therapy for either sorafenib or everolimus (IGR). They concluded that SGR evaluation revealed: 1) better evaluation of tumor response, regardless of RECIST criteria, 2) had independent prognostic value, 3 ) the possibility that continuation of sorafenib or everolimus after disease progression might be beneficial to patients by sustaining a continued suppression of tumor growth.

The following section presents a model of tumor growth rate expressed as an executable algorithm in the form of an Apple App that quantitates subtle changes of tumor specific growth rate (SGR). 


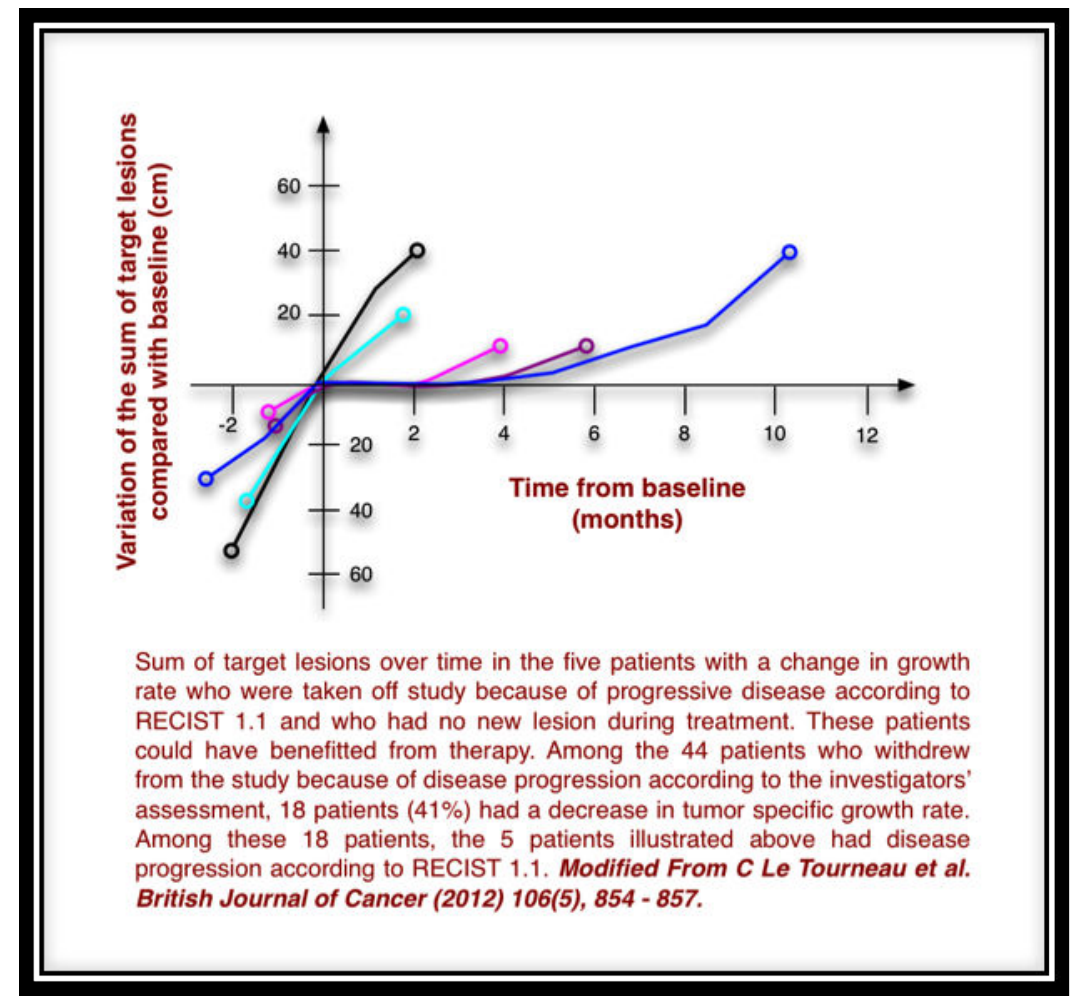

Figure 9. Following a patient's tumor size often reveals subtle changes in the slope of the tumor measurement or marker growth curve as revealed above. These subtle changes in growth rate are not associated with a significant decrease of tumor size or marker value. As Le Tourneau et al. and Ferte et al. demonstrate, subtle changes in tumor growth rate are not evaluated as a response when applying RECIST 1.1 criteria nevertheless, they do represent a true cytostatic effect of targeted therapies that may translate into a meaningful prolonged survival.

\section{SGR is a useful tool to identify subtle drug-associated tumor or marker kinetic changes of tumors}

Mehrara, as part of his PhD thesis at the Department of Radiation Physics, University of Gothenburg, Goteborg, Sweden presented an analysis of tumor growth kinetics based on the tumor specific growth rate constant (SGR). The analysis assumes that for most practical purposes clinically observable tumor growth follows exponential growth. Additionally, this is true for the surrogate PSA tumor marker. SGR is rapidly calculable by hand-held mobile devices and facilitates the rapid identification of tumor responses easily overlooked in the clinic, many of which are not readily apparent without computer analysis. Occasionally, changes of SGR uncover subtle tumor stimulation. 
Construction of the exponential growth curve, similar in shape to the mid portion of the Gompertzian curve Figure 1, requires just two different measurements of tumor volume (or diameter, area, cell number) or a surrogate marker at two different times to satisfy the exponential growth equation: $V t=V 0 e^{a t}$. Here " $\alpha$ " is the exponential growth constant, and $\mathrm{V}_{\mathrm{t}}$ and $V_{0}$ are the tumor volume at times $t$ and $t_{0}$, respectively. This model implies that tumor volume can increase indefinitely and the growth rate of a tumor is proportional to its volume and $\mathrm{dV} / \mathrm{dt}=\mathrm{aV}$.

SGR is the relative change in tumor volume per unit time calculable as percent increase or decrease of tumor volume per unit time. Excluding mutations, for exponentially growing tumors, SGR is constant, i.e., SGR or $\alpha$ is independent of tumor volume or age. Faster growing tumors have higher SGR values, SGR=0 represents non-growing tumors; a negative SGR represents tumor regression. In 1956 Collins et al. [9] graphically introduced the concept of tumor doubling time. The DT formulation was proposed in 1961 [10]: DT $=\left(\mathrm{t}_{2}-\mathrm{t}_{1}\right) * \ln (2) /$ $\left[\ln \left(\mathrm{V}_{2} / \mathrm{V}_{1}\right)\right]$. Other relationships of importance include the specific growth rate, SGR = $\ln \left(V_{2} / V_{1}\right) /\left(t_{2}-t_{1}\right)$ and DT $=\ln (2) / S G R$. These equations are descendants of the primary exponential growth equation, $\mathrm{V}_{\mathrm{t}}=\mathrm{V}_{0} \mathrm{e}^{\text {at }}$

Mehrara expresses concerns based on his mathematical treatment of SGR and DT suggesting that for clinical studies, SGR is the best indicator of tumor growth. Tumor growth rate, especially but not limited to urology circles, is usually quantified as DT i.e. PSA-DT. Because of the subtle mathematical relationship between SGR and DT, use of DT alone to evaluate therapeutic effects may give erroneous results.

Mehrara's studies revealed that DT has several drawbacks when used to describe tumor or tumor marker growth rates. The shortfalls include 1) for brief measurement time intervals, or high volume and very small measurement uncertainties the mean DT can either overestimate or underestimate the average growth rate; 2) DT approaches infinity for very slow growing tumors and is mathematically limited while SGR is a continuous variable no mater the speed and 3) the non normal frequency distribution of DT values restricts use of parametric statistics thus reducing use of more discriminatory statistics especially when studying small samples [77]. Unlike DT, SGR is definable for all tumor volume changes no matter how small, and it is Gaussian (normally) distributed allowing use of parametric statistics. SGR is more accurate to use when considering growth fraction, cell loss rate, and tumor growth rate heterogeneity. For these reasons, Mehrara opines that SGR be used instead of DT, to quantify tumor growth rate.

Accuracy and clinical outcome analysis comparing SGR and DT would be a valuable area of research in light of the cytostatic changes leading to subtle changes of growth rate characteristic of targeted therapies. Later, an in depth illustration of the differences between DT and SGR will help illuminate this issue.

Collins and Schwartz $[9,10]$ both analyzed several tumors in patients as they defined the use of tumor volume doubling time. Note that for bronchogenic lung cancers a semi-logarithmic plot of tumor diameter (y-axis) versus a linear time period ( $\mathrm{x}$-axis) produces a near straight line Figure 2. 


\section{Measuring tumor growth}

It is imperative to depend on sensitive and precise marker assays. Guess [38] tried to address this problem by use of splines or line segments to average all PSA-DT values in an attempt to better detect therapy-induced changes of PSA-DT. Unfortunately, this computerized technique is cumbrous for most to apply.

The accurate and reproducible measurement of tumor diameters from imaging studies is critical. Keep in mind that occasionally plain radiographs of larger lesions are preferred because CT imaging may slice through a lesion at variable levels producing aberrant results for elliptical lesions.

A closer look at differences between DT and SGR.

The mathematical relationship between DT and SGR as revealed by the exponential growth model is important because as displayed in Figure 10, sole use of tumor volume doubling time (TV-DT) or tumor marker doubling time (PSA-DT) rather than tumor or marker specific growth rate as a measure of treatment outcome may be destined for failure depending on the magnitude of differences in the clinical study. Applying the exponential model of tumor growth to published studies reporting only DT as displayed here Table 1,2 and Figures 11,12 reveals discordant conclusions from those using SGR. Note that the DT is mathematically logarithmically related to the inverse of the exponential growth constant (SGR): SGR = $\ln (2) / \mathrm{DT}$.

The opposite results using SGR compared to those obtained with DT are critical since prostate cancer research is steeped in the use of the PSA-DT to predict survival, tumor dissemination, relapse, and tumor response to drugs and hormones and to radiation efficacy. In the prostate cancer literature use of DT as a parameter of response is established canon.

Mehrara reveals that DT is not normally symmetrically distributed (non-Gaussian distribution) and its use as an indicator of treatment response could yield inaccurate conclusions. Changes in DT over-predict drug effects in slow growing tumors while they under-predict in rapidly growing tumors and DT is essentially of no value for tumor volumes (or markers) that show no change in value (stable disease) where DT approaches infinity see Figure 10.

Work by others confirms the importance of the tumor or marker-specific growth rate. Stein et al. [46] studied a combination of equations that simultaneously modeled both tumor/ PSA regression and tumor/PSA exponential growth. They found that only the exponential growth equation with its specific growth rate constant (PSA-SGR) predicted a statistically significant high mortality hazard ratio of 5.14 (95\% confidence interval, 3.10 - 8.52) in his study group of patients with prostate cancer. The disease regression formula was unable to predict patient mortality. 


\section{Why PSA-SGR is more useful than PSA-DT}

As noted in Figure 10, when SGR is fast and increases 1\% from 4 to 5\%/day, the doubling time changes 1.3-fold from 4 to 3 days (a slight change). However, when the SGR is slower and increases $1 \%$ from $1 \cdot 2 \% / d$, doubling time changes four-fold from 69 to 17 days (a large change). A DT of 1-day does not represent the same growth rate when the tumor is slowing as when the tumor is rapidly growing. As the absolute value of SGR approaches zero, DT approaches infinity and is of no practical use other than to say the tumor or marker is stable. Because of the DT-SGR relationship at the extremes of tumor or marker growth, therapy-induced changes in doubling times at the extremes of SGR do not accurately represent the magnitude of the impact of therapy.

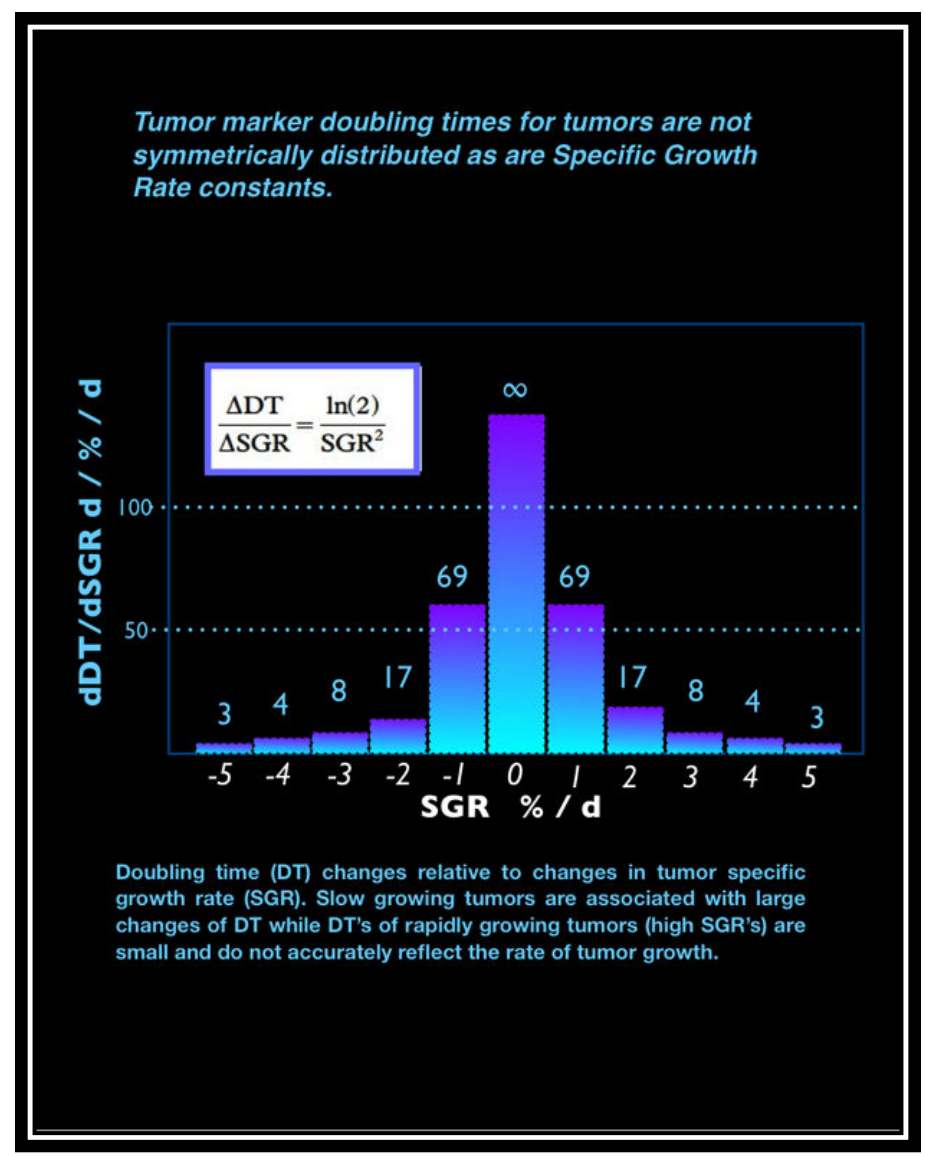

Figure 10. This figure, modified from Mehrara [70], displays the variation of tumor volume doubling time or tumor marker doubling time (DT) per unit change of tumor specific growth rate (SGR) based on: 


\section{Clinical application of DT and SGR: Discordant results}

Mehrara retrieved data from two previously published clinical studies [70]. The first by Guess et al. [38] Table 1 who studied the effect of modified citrus pectin (MCP) on PSA-DT of 12 prostate cancer patients. Mehrara extracted data and analyzed for both PSA-DT and PSA-SGR before and after therapy. The difference between PSA-DT before and after treatment was not found to be statistically significant by the paired t-test $(p=0.27)$. Nevertheless, when transforming PSA-DT to PSA-SGR the difference before and after MCP treatment is statistically significant by the paired t-test $(p=0.003)$ and nonparametric Wilcoxon matched pairs signed rank test: $p=0.002$. Thus, a therapy initially deemed ineffective by PSA-DT analysis, when analyzed for a group of patients based on PSA-SGR proved to be highly significant Table 1.

\begin{tabular}{|c|c|c|c|c|}
\hline \multicolumn{5}{|c|}{ Effect of modified citrus pectin (MCP) on PSA-DT and PSA-SGR } \\
\hline Patient & $\begin{array}{c}\text { Before Rx } \\
\text { PSA-DT } \\
\text { (mo) }\end{array}$ & $\begin{array}{c}\text { After Rx } \\
\text { PSA-DT } \\
\text { (mo) }\end{array}$ & $\begin{array}{l}\text { Before Rx } \\
\text { PSA-SGR (\%/mo) }\end{array}$ & $\begin{array}{l}\text { After Rx } \\
\text { PSA-SGR (\%/mo) }\end{array}$ \\
\hline$A$ & 3.97 & 13.34 & 17.46 & 5.16 \\
\hline B & 5.67 & 10.11 & 12.22 & 6.86 \\
\hline$C$ & 1.14 & 2.91 & 60.80 & 23.82 \\
\hline$D$ & 3.37 & 7.71 & 20.57 & 8.99 \\
\hline$E$ & 1.58 & 16.49 & 43.87 & 4.20 \\
\hline $\mathrm{F}$ & 10.5 & 7.97 & 6.60 & 8.70 \\
\hline G & 2.66 & 11.95 & 26.06 & 5.80 \\
\hline $\mathrm{H}$ & 3.64 & 3.27 & 19.04 & 21.20 \\
\hline I & 2.04 & 4.96 & 33.98 & 13.97 \\
\hline J & 2.33 & 3.24 & 29.75 & 21.39 \\
\hline $\mathrm{K}$ & 6.29 & -155.49 & 11.02 & -0.45 \\
\hline$L$ & 5.12 & -645.51 & 13.54 & -0.11 \\
\hline \multicolumn{5}{|c|}{$\begin{array}{l}\text { Nonparametric Wilcoxon matched pairs signed Nonparametric Wilcoxon matched pairs signed } \\
\qquad \text { rank: } p=0.42 \quad \text { rank: } p=0.002\end{array}$} \\
\hline
\end{tabular}

Table 1. Guess et al. [38] studied the effect of modified citrus pectin (MCP) on PSA-DT of 12 prostate cancer patients. Mehrara extracted that data and analyzed both PSA-DT and PSA-SGR before and after therapy. The difference between PSA-DT before and after treatment was not statistically significant by the paired t-test $(p=0.27)$. Nevertheless, when transforming PSA-DT to PSA-SGR the difference before and after MCP treatment is statistically significant by the paired t-test $(p=0.003)$ and nonparametric Wilcoxon matched pairs signed rank test: $p=0.002$.

A second analysis of original data by Nishida et al. (1999) [78] was based on a study of the correlation of tumor volume and the CA19-9 tumor marker of pancreatic cancer patients Ta- 
ble 2. The correlation between CA19-9-DT and tumor volume-DT was statistically significant $(\mathrm{p}<0.0001)$. However, after converting tumor-volume-DT to TV-SGR and CA19-9-DT to CA19-9-SGR, correlation between CA19-9-SGR and TV-SGR was no longer statistically significant ( $>0.3$ ). Since SGR is the preferred parameter, the initial analysis of Nishida may benefit from a second look.

\begin{tabular}{|c|c|c|c|c|}
\hline \multicolumn{5}{|c|}{ Relationship between CA19-9-DT and TV-DT vs. CA19-9-SGR and TV-SGR } \\
\hline Patient & $\begin{array}{l}\text { CA19-9 DT } \\
\text { (Days) }\end{array}$ & $\begin{array}{l}\text { Tumor-DT } \\
\text { (Days) }\end{array}$ & $\begin{array}{c}\text { CA19-9-SGR } \\
\% / \text { day }\end{array}$ & $\begin{array}{c}\text { Tumor-SGR } \\
\% / \text { day }\end{array}$ \\
\hline A & 8.3 & 34.8 & 8.4 & 2 \\
\hline B & 39.7 & 44.6 & 1.7 & 1.6 \\
\hline C & 46.3 & 34.5 & 1.5 & 2 \\
\hline $\mathrm{D}$ & 36.5 & 21.2 & 1.9 & 3.3 \\
\hline E & 30.4 & 47.7 & 2.3 & 1.5 \\
\hline $\mathrm{F}$ & 67.1 & 112.8 & 1 & 0.6 \\
\hline G & 44.7 & 70.6 & 1.6 & 1 \\
\hline $\mathrm{H}$ & 24.7 & 18.4 & 2.8 & 3.8 \\
\hline । & 42.7 & 50.6 & 1.6 & 1.4 \\
\hline J & 137.5 & 231.6 & 0.5 & 0.3 \\
\hline \multirow[t]{2}{*}{ K } & 42.3 & 39.3 & 1.6 & 1.8 \\
\hline & \multicolumn{2}{|c|}{ Linear regression: $r^{2}=0.89$} & \multicolumn{2}{|c|}{ Linear regression: $r^{2}=0.09$} \\
\hline
\end{tabular}

Table 2. This table displays the extracted data from Nishida's study [78] of the correlation of tumor volume and the CA19-9 tumor marker of pancreatic cancer patients. The correlation between CA19-9-DT and tumor volume-DT was statistically significant $(p<0.0001)$. However, after converting tumor-volume-DT to TV-SGR and CA19-9-DT to CA19-9SGR, correlation between CA19-9-SGR and TV-SGR was no longer statistically significant $(p>0.3)$.

Most prostate cancer studies employ changes in the PSA-DT. PSA-DT values are not normally distributed and thus not readily subject to more sensitive parametric statistical analysis. However, PSA-specific growth rate is normally distributed and parametric statistics can be applied. Nonparametric statistical methods lose discriminatory power especially for clinical studies of smaller groups of patients [77].

During a cursory review of the literature we found two additional studies, one dealing with the effects of celecoxib on PSA-DT Figure 11 and the other investigating the effects of a combination of calcitriol and naproxin on PSA-DT of prostate cancer patients Figure 12.

Smith et al. [79] Figure 11 studied the biologic activity of celecoxib, a selective cyclooxygenase-2 inhibitor, in men with recurrent prostate cancer using change in PSA-DT as the primary outcome variable. We carefully extracted the data from his graphic report. We applied the Wilcoxon matched-pairs signed rank test [two tailed] (for nonparametric distribution of 
PSA-DT) to the data. PSA-DT before versus after celecoxib was highly significant: $p=0.0006$. After transformation of PSA-DT to PSA-SGR, the Paired t-test [two tailed] for parametric distribution of PSA-SGR suggests that the celecoxib effect lacked statistical significance $p=0.213$ !

A second study by Srinivas [80] Figure 12 evaluated naproxen in combination with calcitriol in patients with early recurrent prostate cancer. All patients received $45 \mu \mathrm{g}$ of calcitriol (DN101, Novacea, South San Francisco, CA, USA) orally once a week with naproxen 375 mg twice a day and were evaluated for a biochemical PSA response and a change in PSA doubling time (PSA-DT). Testing the efficacy of the combination therapy using changes of PSADT by the non-parametric Wilcoxon matched-pairs signed rank test [two tailed] $p=0.037$ a significant difference. However, after transforming PSA-DT to PSA-SGR $\left(\mathrm{SGR}_{\mathrm{PSA}}=\ln (2) /\right.$ $\left.\mathrm{DT}_{\mathrm{PSA}}\right)$, analysis with the parametric Paired t-test [2-tailed] indicate naproxen plus calcitriol was not effective in slowing tumor growth, $\mathrm{p}=0.213$.

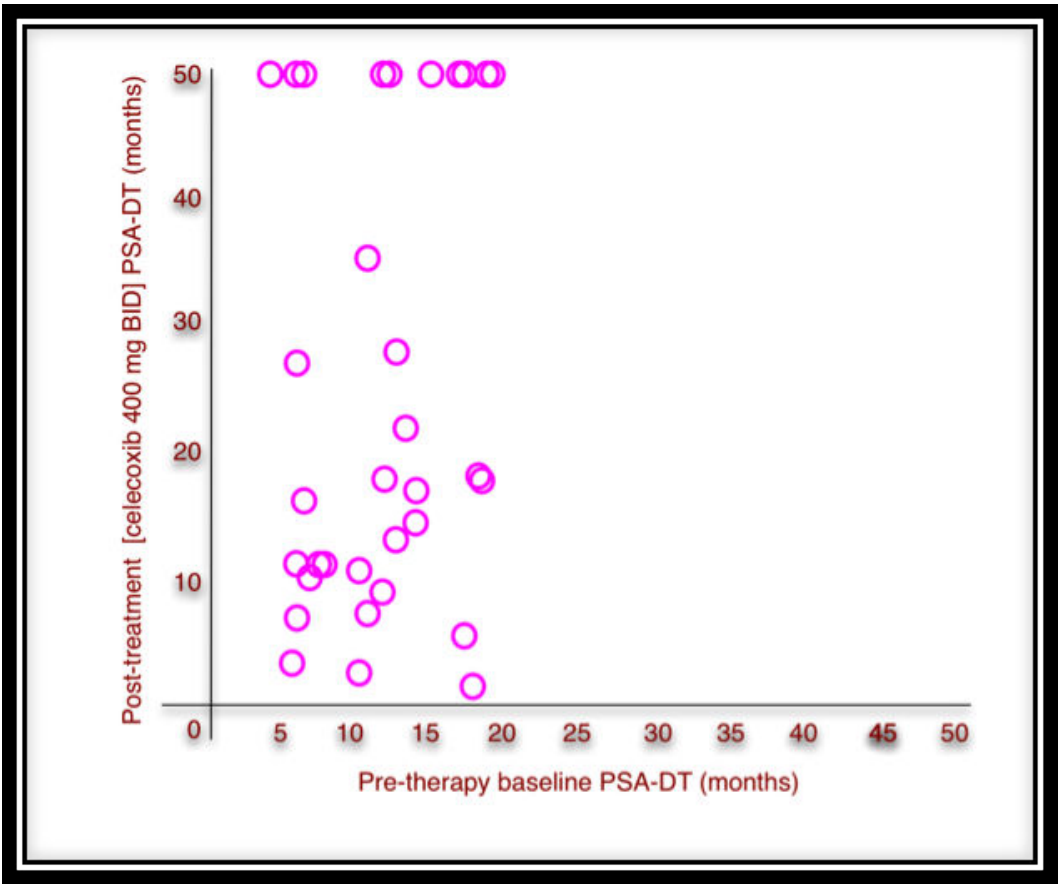

Figure 11. Smith et al. [79] studied the biologic activity of celecoxib, a selective cyclooxygenase-2 inhibitor, in men with recurrent prostate cancer using change in PSA-DT as the primary outcome variable. We retrieved their graphic data for our own analysis. A histogram of the PSA-DT paired differences for before and after celecoxib appears normally distributed. Applying the parametric Paired t-test statistic for significance of the difference yields $p=0.0002$. Next, we transformed the same (before-after celecoxib PSA-DT data with to PSA-SGR before and after pairs and applied the paired t-test. Contrary to the statistical analysis for celecoxib induced change of PSA-DT, changes of PSA-SGR revealed that the celecoxib difference was no longer significant, $p=0.213$ ! 


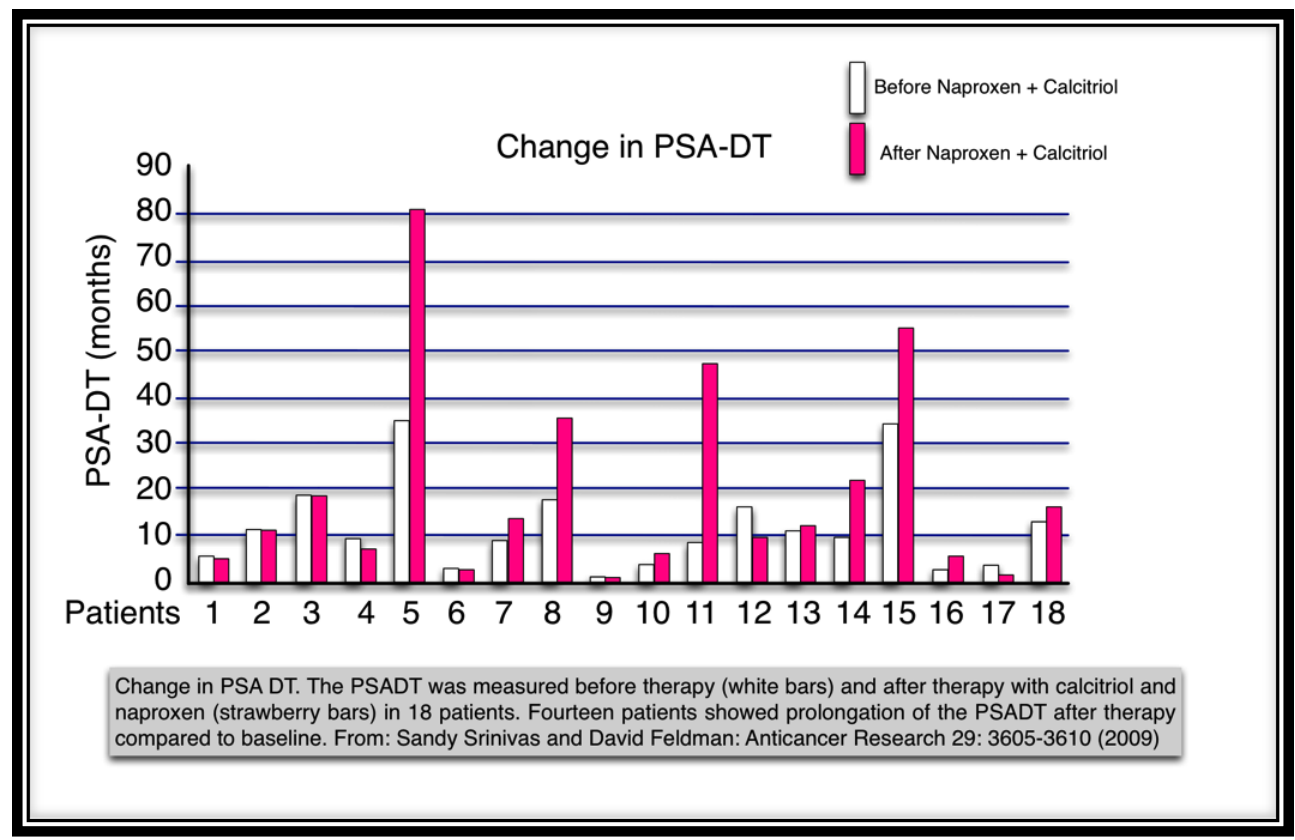

Figure 12. Sinivras and Feldman [80] evaluated naproxen in combination with calcitriol in patients with early recurrent prostate cancer. All patients received $45 \mu \mathrm{g}$ of calcitriol (DN101, Novacea, South San Francisco, CA, USA) orally once a week with naproxen $375 \mathrm{mg}$ twice a day and were evaluated for a biochemical PSA response and a change in PSA doubling time (PSA-DT). Applying the paired t-test for statistical significance (before PSA-DT and after PSA-DT) resulted in $p=0.034$. Nevertheless, after transforming PSA-DT to PSA-SGR (PSA-SGR $=\ln (2) / P S A-D T)$, analysis with the paired t-test [2-tailed] suggested naproxen plus calcitriol was not effective in slowing tumor growth, $p=0.213$.

The non-linear relationship between the SGR and DT may be responsible for erroneous interpretations of treatment effects reported in prior prostate cancer trials that published results solely in terms of changes in PSA-DT Figure 10.

\section{Evaluation of tumor and surrogate marker drug responses, rate of change of response:
$S G R$ acceleration $=(S G R$ after $R x-S G R$ before $R x) /(t 2-t 1) ;$ A positive number

The dynamic of PSA change was used as an early predictor of overall survival after a short exposure to docetaxel therapy (4 doses). Knowledge that a drug may extend survival after just a short exposure would minimize toxicity from ineffective drugs. Hannenin's work [81] found that a rapid rate of PSA decline expressed as PSA half-life $<70$ days was associated with a longer overall-survival Figure 13. This result was independent of other known markers of survival and allowed for a greater survival differentiation than PSA suppression 
alone. Response-time evaluations may play a new role in determining drug efficacy earlier than usual. I would propose study of an alternate expression for tumor acceleration or deceleration in terms of SGR as: SGR (accel...decal) = SGR2-SGR1/( $\left.t_{2}-t_{1}\right)$. The value of this expression may be positive for acceleration or negative for deceleration.

De Crevoisier [82] found that a PSA decline 6 weeks after the start of EBRT when used as monotherapy and 3 months after the start of androgen deprivation therapy (ADT) in patients treated with combined ADT and external beam radiation is predictive of progression and specific survival.

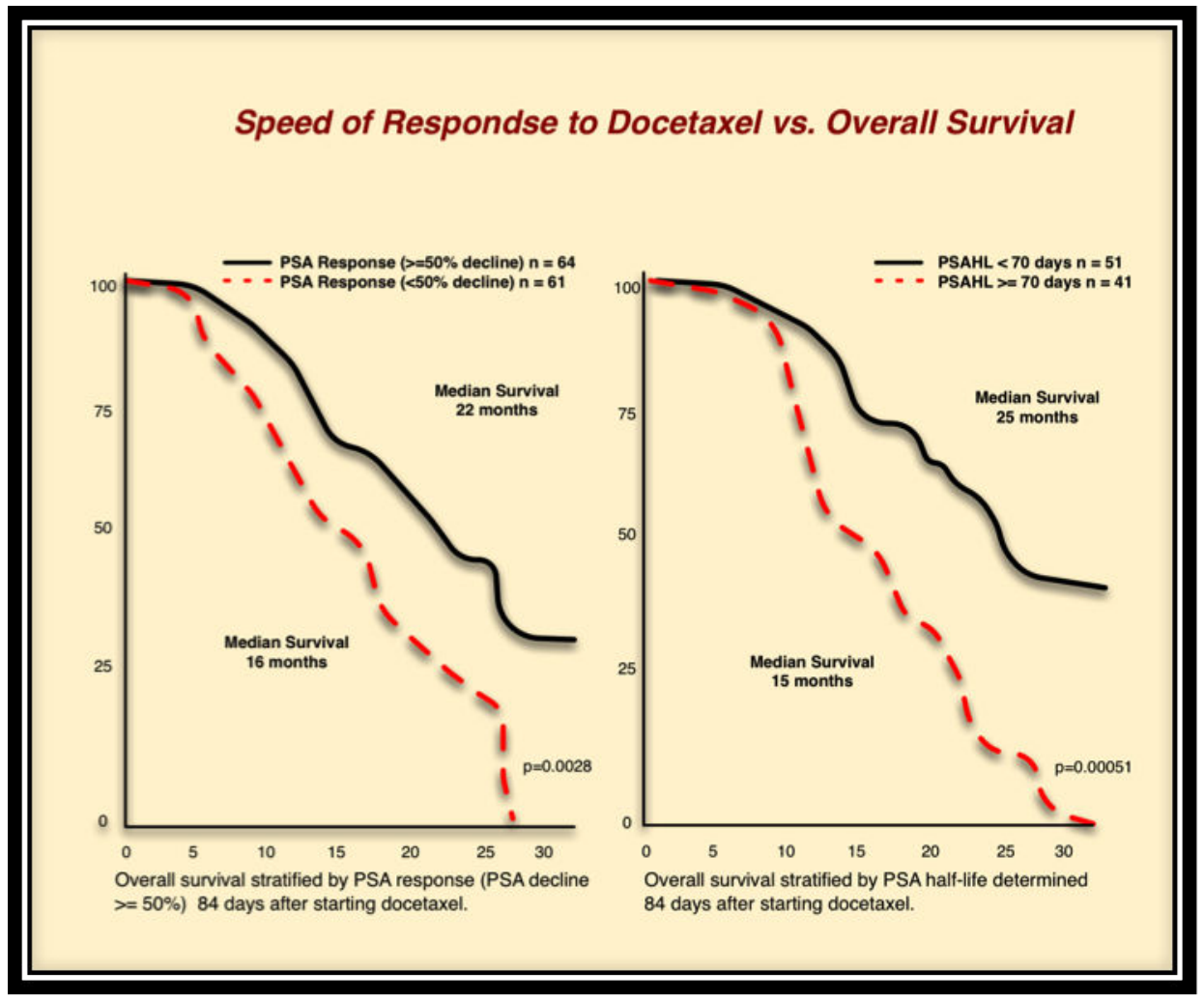

Figure 13. Treatment-associated tumor/marker deceleration in response to docetaxel. The magnitude of rate of change (acceleration-deceleration) of SGR resulting from therapy is an early predictor of prostate-specific survival.

Figure 14 illustrates a computer analysis of a prostate cancer patient treated with docetaxel. A pelvic node is noted to grow over 4.5 months from 1.3 to $1.6 \mathrm{~cm}$ in greatest dimension. This establishes the pre-therapy SGR of $0.46 \% / \mathrm{d}$ and the tumor volume (assuming a sphere) before starting therapy is $2.1 \mathrm{cc}$. Fifty-one days of therapy induces a decrease of tumor diameter to 0.9 $\mathrm{cc}$ and a decrease of tumor volume to $0.38 \mathrm{cc}$. Had the tumor grown uninterrupted the project- 
ed tumor volume would have been $2.7 \mathrm{cc}$. In this case, the value for deceleration of SGR for the tumor: is given as (SGR2 after $R x-S G R 1$ before $R x) /(t 2-t 1)=-0.021 \% / d / d$.

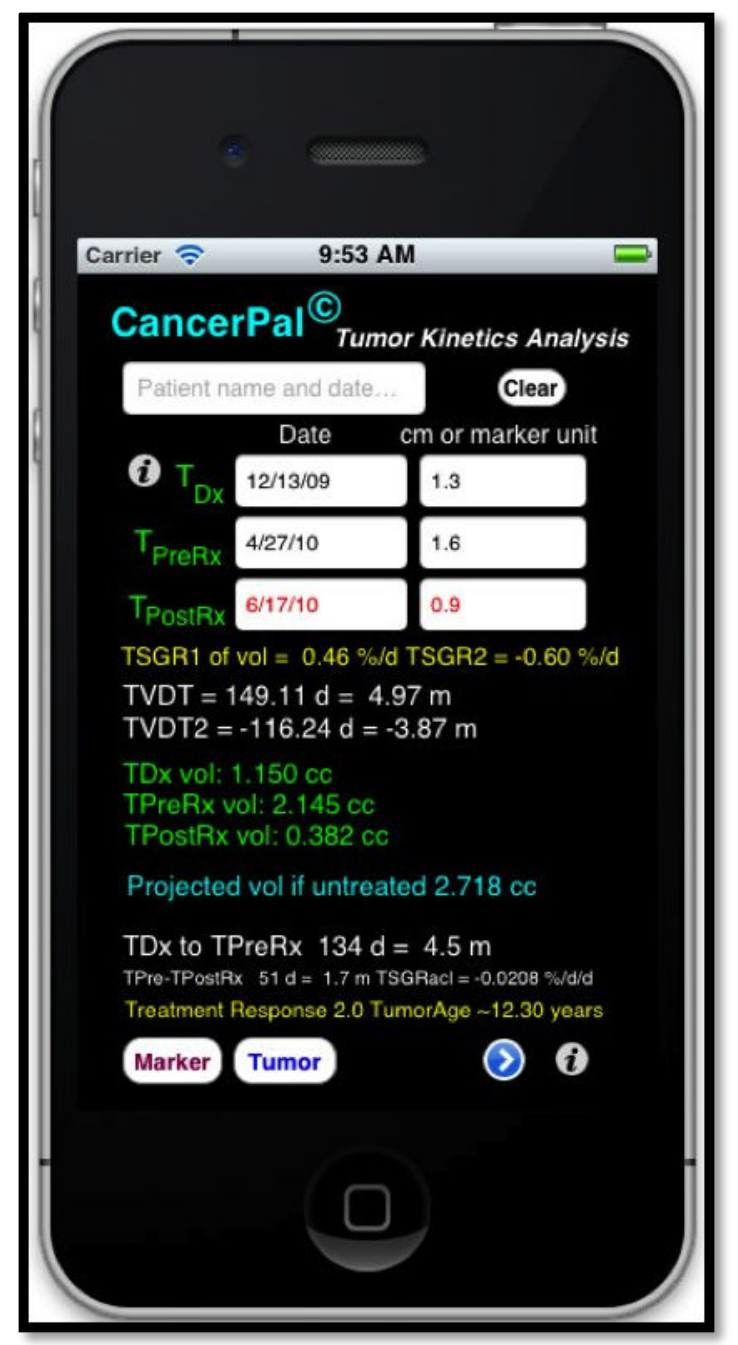

Figure 14. This calculation displays results for a patient treated with docetaxel (see text).

This is an objective measure of the rate of change of SGR. The treatment response is displayed as $=+2.0$. This assigns a calculated continuous variable as a measure of the degree of response and is used to objectively compare docetaxel efficacy to any other administered drug. Positive TR values represent tumor reduction compared to the projected tumor size while a negative TR represents tumor growth relative to the projected size. Estimated age of 
tumor, here approximated $~ 12.3$ years, is a calculated value based on the initial SGR of $0.46 \% / \mathrm{d}$ in the absence of therapy. This assumes constant, continuous exponential growth over many years. Tumor age calculations are gross approximations and notoriously subject to large error.

Figure 15 illustrates the evaluation for a 68 year-old man undergoing watchful waiting for a Gleason score $3+3=6$, T1c prostate cancer. Three PSA values are displayed for three sequential dates. When the patient was asked if he had changed medication between $3 / 2 / 11$ and 5/1/11 he noted he was ingesting a new Chinese herbal mixture sold to enhance energy and libido.

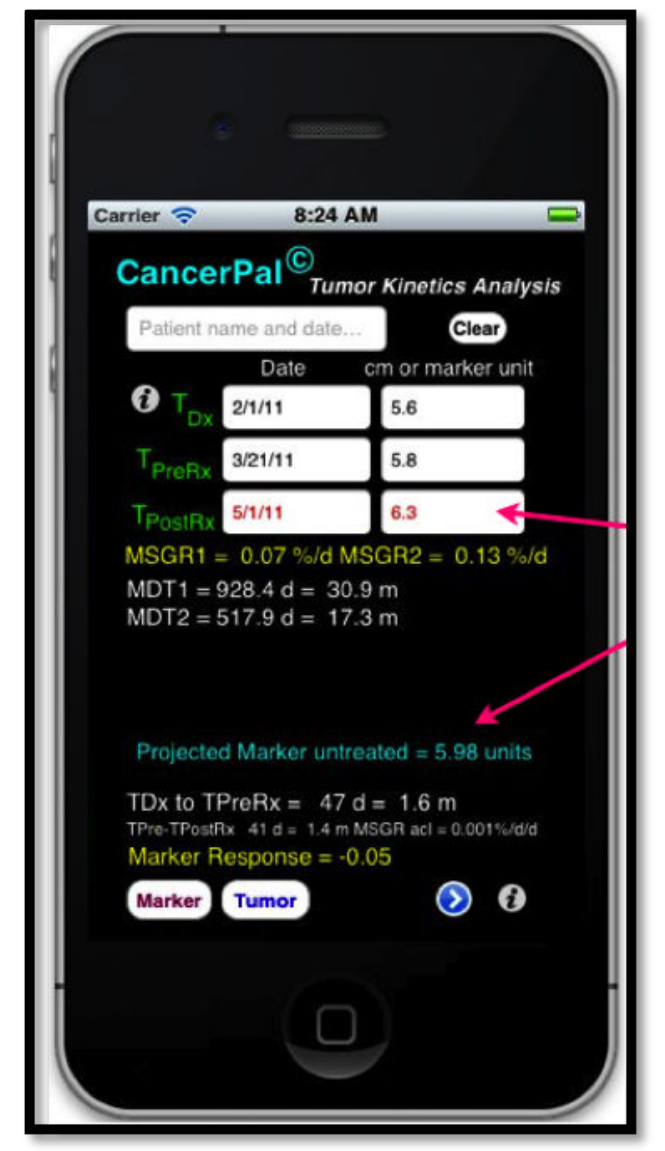

Figure 15. Evaluation of a 68 year-old man undergoing watchful waiting for a Gleason score $3+3=6, T 1 c$ prostate cancer. The patient was ingesting a stimulatory Chinese herb. 
Subtle acceleration of the tumor marker value was uncovered by inspection of the projected PSA value for 5/1/11 as compared to the actual measured value for that date. Notice the confirmative quantitative measures given by the calculation Figure 14 of the marker specific growth rate $\mathrm{MSGR}_{2}=0.13 \% / \mathrm{d}$ compared to $\mathrm{MSGR}_{1}=0.07 \% / \mathrm{d}$; marker doubling time MDT2 $=17.3$ months compared to the initial $\mathrm{MDT}_{1}=30.9$ months; by both the positive value for MSGR acceleration $=+0.001 \% / \mathrm{d} / \mathrm{d}$ and by the negative value for marker response $\mathrm{MR}=-$ 0.05. Based on the marker-specific growth rate (MSGR) for the first interval TDx thru TPRx (the date at initiation of therapy) of $0.07 \% / \mathrm{d}$, the App calculated the expected PSA on 5/1/11 to be $5.98 \mathrm{ng} / \mathrm{ml}$. However, the measured value was higher $=6.3 \mathrm{ng} / \mathrm{ml}$. The negative value for MR of -0.05 indicates a negative marker response thus PSA expansion (marker acceleration confirmed this $=+0.001 \% / \mathrm{d} / \mathrm{d}$ ). We suspected that the Chinese herb might have caused subtle acceleration of PSA production and or tumor growth. Other explanations for acceleration of the PSA value include decreased clearance of PSA or the subtle appearance of a mutated, faster growing clone of PSA-producing tumor cells. Note that in the absence of knowledge of the inherent initial PSA-SGR between 2/1/11 and 3/2/11 and calculation of the expected projected value of PSA for 5/1/11, the subtle PSA acceleration would have been missed.

\section{Predicting approximate tumor size or marker value for any arbitrary date in the future}

Assuming untreated clinical cancers and their markers expand at a relatively constant exponential rate, it is possible to predict values for tumor diameter, volume and marker for any arbitrary future date. Figure 16 displays a PSA projection made for a patient with newly diagnosed prostate cancer who asked if a preplanned three-month holiday before initiation of therapy could jeopardize his chance for a curative procedure. The prediction, assuming constant exponential expansion of serum PSA, is that the PSA value upon returning from sabbatical would increase from 9.4 to $16.28 \mathrm{ng} / \mathrm{ml}$. This alarmed the patient and he cancelled the trip to initiate therapy.

\section{Unique treatment paradigms may be suggested by analysis of tumor growth rate}

Figure 17 illustrates results for a patient with pancreatic cancer post Whipple procedure who was found on 6/4/10 to have an enlarged peri-aortic mass $=1.8 \mathrm{~cm}(3.1 \mathrm{cc})$. Repeat CT on $8 / 27 / 10$ noted increased size to $2.9 \mathrm{~cm}(12.8 \mathrm{cc})$. Therapy with gemcitabine was initiated on $8 / 27 / 10$. Post therapy reevaluation of the mass on $12 / 24 / 10$ revealed growth to $3.1 \mathrm{~cm}$ $(15.6 \mathrm{cc})$. The patient was discouraged and frightened and thought he had wasted precious 


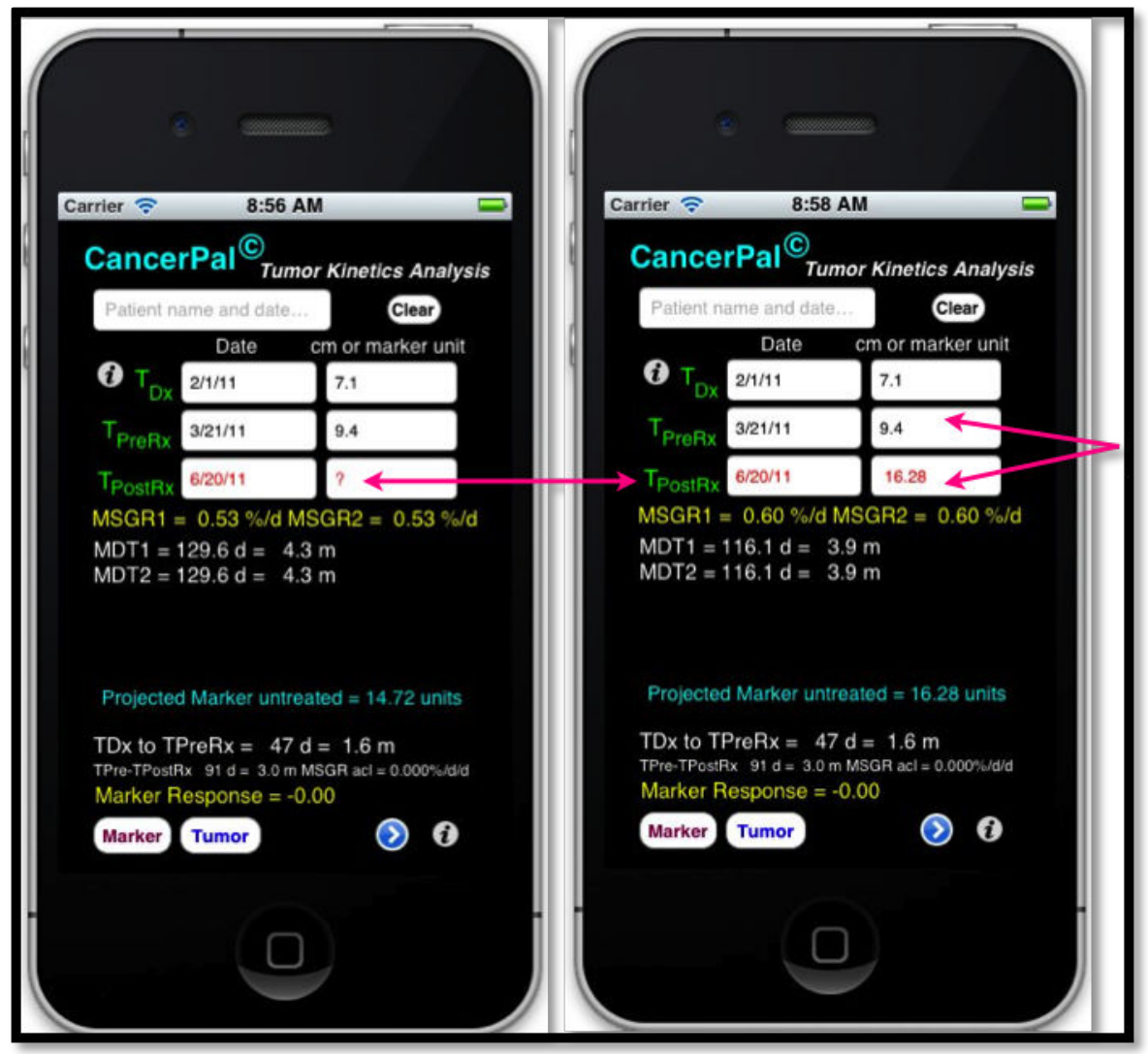

Figure 16. PSA projection made for a patient with newly diagnosed prostate cancer

time and subjected himself to undo toxicity for no gain. However, evaluation revealed that had the tumor never been treated with gemcitabine it would have reached the projected volume of 96.9 cc by $12 / 24 / 10$. Thus, based on the initial exponential growth rate from $6 / 4 / 10$ thru $8 / 27 / 10$, the tumor volume was actually $84 \%$ less than what it would have been had no drug been given ( 15.6 cc vs. 96.9 cc).

This patient experienced substantial tumor suppression by gemcitabine in spite of its growth. Under these circumstances, when there are poor second choices for effective therapy, instead of discarding gemcitabine, perhaps addition of another compound with differing toxicity might be a reasonable option. 


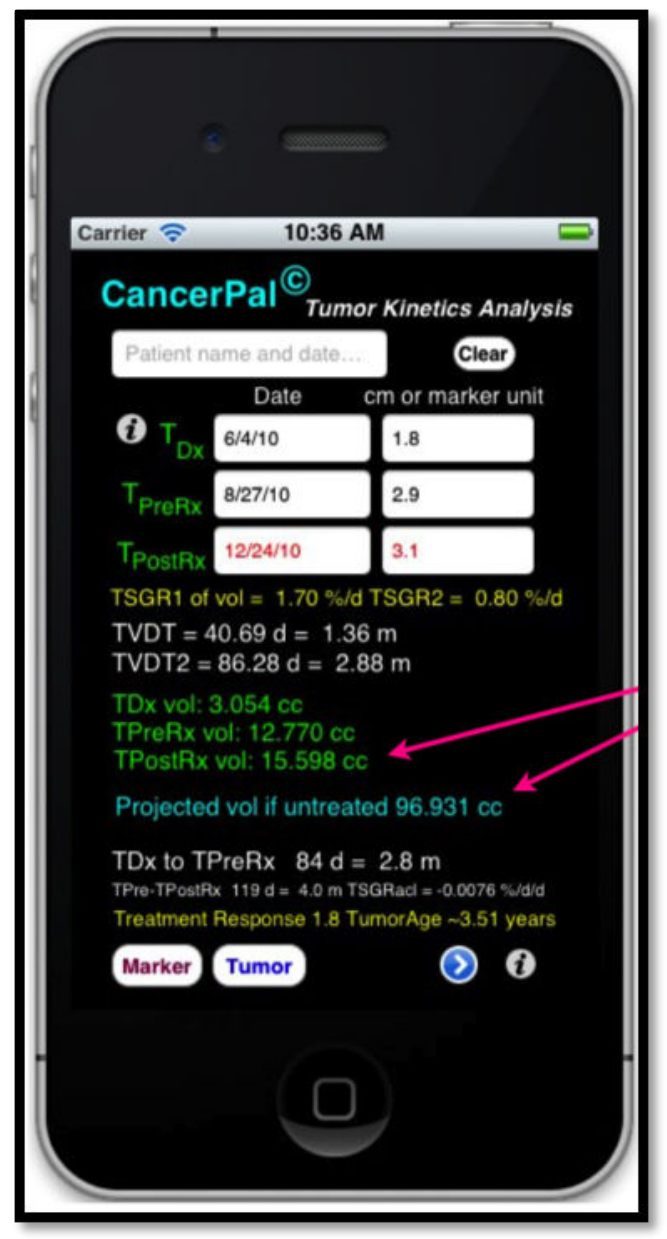

Figure 17. The tool we developed to facilitate calculation of tumor kinetics is named CancerPal ${ }^{\circ}$. The software App is available from Apple Corporation's App store. The App analyzes kinetic changes of tumor markers and or tumor diameter/volume/area and is run on the iPhone, iPad, or iPod. Clinical use is facilitated by the small size and portability of the new hand-held devices. The App is routinely used in our clinic for objectively measuring subtle drug effects on tumor and the dynamics of surrogate tumor markers. A video tutorial of the App is available at www.healthsciencereports.com.

\section{Conclusion}

Several principles of prostate cancer management rely on the absolute and dynamic values of various formulations of PSA i.e. PSA-V, PSA-DT and PSA-SGR. This review introduces SGR, a parameter that is underused and closely reflects the true growth rate of tumors un- 
dergoing exponential expansion. Several instances are presented where results of studies employing PSA-DT yield statistically divergent results after converting PSA-DT to PSASGR. It is recommended that for some studies results be reevaluated in terms of PSA specific growth rate, PSA-SGR.

Newly introduced targeted therapies require innovative techniques to evaluate drug efficacy. Tumor or tumor marker specific growth rate and the concept of projected tumor or marker value are tools capable of quantitatively evaluating subtle effects of targeted drugs. Calculation of the projected tumor size and tumor marker values is critical to properly evaluate subtle drug-tumor proliferative outcomes.

\section{Appendix}

\section{CancerPal $^{\complement}$}

It is important to realize that $\mathrm{CancerPal}^{\odot}$ remains an experimental tool used strictly for analysis of clinical and laboratory data by cancer researchers, pharmacists or clinical research radiation and medical oncologists. The methods used in designing this tool have been discussed primarily in the references listed below with special attention given to the work of Mehrara et al. PNA, A Limited Liability Corporation, cannot be held responsible for any treatment modifications or recommendations made based on this research tool.

What CancerPal ${ }^{\odot}$ does

$\mathrm{CancerPal}^{\odot}$ evaluates whether a chemotherapy or targeted therapy should be continued alone, possibly dropped or added to by revealing concealed drug activity causing suppression of the tumor specific growth rate. The app uncovers occult efficacy of drugs by comparing the measured drug-induced tumor size vs. the projected tumor size or projected tumormarker value that would occur in the absence of any therapy. Sudden changes in tumor growth rate suggesting drug related tumor stimulation or a detrimental, growth-promoting mutation is rapidly identified. CancerPal ${ }^{\odot}$ may uncover hidden tumor acceleration unexpectedly caused by drugs, immunosuppression or alternative therapies thought to be harmless

CancerPal $^{\odot}$ uses a tumor's specific growth rate (TSGR) defined as percentage increase in volume per day or percentage increase in the specific tumor marker per day thus avoiding errors inherent in the doubling time calculation which consistently overestimates the growth rate of slowly growing tumors and underestimates the growth rate of rapidly growing tumors.

This app predicts the tumor diameter or tumor marker value at any time in the future assuming constant exponential tumor or tumor marker growth over the period of observation. This, when compared to the actual measured tumor diameter or marker value, identifies tumor response, stability or acceleration. The app predicts a tumor marker or diameter at any time point in the future based on patient-specific tumor kinetics. CancerPal ${ }^{\odot}$ may quickly 
alert the clinician of emergence of a mutant, more aggressive, rapidly dividing clone of tumor cells suggesting a review of therapy. Analysis based on continual exponential growth for the relatively short time (several months) in the multi-year history of tumor growth has been found to be more useful for kinetic calculations in spite of some tumors demonstrating Gompertzian growth over the long haul (several years)

Continuous variables for Tumor Response (TR) and Marker Response (MR) allow for quantitation of drug/biological response modulator effects. Negative values of TR and MR indicate tumor acceleration, values close to or equal to zero indicate lack of response while positive values confirm beneficial tumor response. Responses are numerically quantitated and elusive disease stability may now numerically be defined by a continuous variable. Drugs previously thought to be of no value may be found to induce useful and profound disease stability

The software is helpful for those patients followed by watchful waiting/active surveillance for prostate or any other cancer. Prostate tumors changing biological behavior are immediately identified in a quantitative and objective manner by rapidly uncovering changes in PSA kinetics without the errors inherent in the PSA doubling time (PSA-DT) parameter. The software can help determine whether metastectomy is a reasonable treatment modality for some patients with pulmonary metastasis [83].

$\mathrm{CancerPal}^{\odot}$ uses the exponential growth constant as described by John Spratt to extrapolate backwards to approximate the time of tumor initiation in years based on the rate of growth

Patient data required for analysis

Three dates and three associated measurements of a tumor marker or tumor diameter

- TDx (date at diagnosis + marker value or tumor diameter in $\mathrm{cm}$ )

- TPreRx (date of initiation of $\mathrm{Rx}+$ marker value or tumor diameter in $\mathrm{cm}$ )

- TPostRx (date of measurement of drug effect + tumor marker value or diameter in $\mathrm{cm}$ ).

CancerPal ${ }^{\odot}$ information output:

- Tumor Specific Volume Growth Rates for two intervals (TSGR and TSGR $_{2}$ )

- Tumor Marker Specific Growth Rates for two intervals $\left(\mathrm{MSGR}_{1}\right.$ and $\mathrm{MSGR}_{2}$ )

- Tumor Specific Growth Rate acceleration and deceleration

- Tumor Volume Doubling Times for two intervals (TVDT)

- Tumor Marker Doubling Time for two intervals (MDT)

- Projected TSGR and MSGR at any user designated time in the future

- Treatment Response as both Tumor Response and Tumor Marker Response, both as continuous numerical values used to quantitate the effect of therapy. Negative numbers reveal growth acceleration; values of zero reveal no effect and positive values indicate varying degrees of therapeutic efficacy. 
- Tumor volumes in cc are calculated for TDx, TPreRx and TPostRx.

- Extrapolates back to the time of tumor initiation thus calculating how long it took for the tumor to reach the initial tumor diameter.

- Calculates approximate time to death in the absence of therapy, assuming constant tumor growth rate.

\section{Acknowledgements}

Peter Jahn, Whittier, CA for manuscript review and discussion, mathematical suggestions and statistical considerations.

\section{Author details}

Glenn Tisman

Whittier Cancer Research Building, Whittier, CA, USA

\section{References}

[1] Bassukas ID. Comparative gompertzian analysis of alterations of tumor growth patterns. Cancer Res 1994, Aug 15; 54(16): 4385-92.

[2] Demicheli R, Foroni R, Ingrosso A, Pratesi G, Soranzo C, Tortoreto M. An exponential-gompertzian description of lovo cell tumor growth from in vivo and in vitro data. Cancer Res 1989, Dec 1; 49(23): 6543-6.

[3] Norton L. A gompertzian model of human breast cancer growth. Cancer Res 1988, Dec 15;48(24 Pt 1):7067-71.

[4] Mottram JC. On the correlation between malignancy and the rate of growth of tar warts in mice. Cancer Res 1934, Dec; 22(4): 801-30.

[5] Mottram JC. A further consideration of the growth rates of tar warts in mice and of their autografts. The American Journal of Cancer 1936; 28(1): 115-20.

[6] Laird AK. Dynamics of normal growth. Annual Report 1964; 6971:52.

[7] Laird AK. Dynamics of tumour growth: Comparison of growth rates and extrapolation of growth curve to one cell. Br J Cancer 1965; 19(2): 278.

[8] Friberg S, Mattson S. On the growth rates of human malignant tumors: Implications for medical decision-making. J Surg Oncol 1997, Aug; 65(4): 284-97. 
[9] Collins VP, Loeffler RK, Tivey H. Observations on growth rates of human tumors. Am J Roentgenol Radium Ther Nucl Med 1956, Nov; 76(5): 988-1000.

[10] Schwartz M. A biomathematical approach to clinical tumor growth. Cancer 1961; 14:1272-94.

[11] Spratt JS, Spjut HJ, Roper CL. The frequency distribution of the rates of growth and the estimated duration of primary pulmonary carcinomas. Cancer 1963, Jun; 16:687-93.

[12] Spratt Jr JS, Spratt TL. Rates of growth of pulmonary metastases and host survival. Ann Surg 1964; 159(2): 161.

[13] Steel GG, Lamerton LF. The growth rate of human tumours. Br J Cancer 1966, Mar; 20(1): 74-86.

[14] Loeb S, Kettermann A, Ferrucci L, Landis P, Metter EJ, Carter HB. PSA doubling time versus PSA velocity to predict high-risk prostate cancer: Data from the Baltimore longitudinal study of aging. Eur Urol 2008, Nov; 54(5): 1073-80.

[15] Tosoian J, Loeb S. PSA and beyond: The past, present, and future of investigative biomarkers for prostate cancer. ScientificWorldJournal 2010; 10:1919-31.

[16] Klotz L, Teahan S. Current role of PSA kinetics in the management of patients with prostate cancer. European Urology Supplements 2006, Apr; 5(6): 472-8.

[17] D'Amico AV, Chen MH, Roehl KA, Catalona WJ. Preoperative PSA velocity and the risk of death from prostate cancer after radical prostatectomy. N Engl J Med 2004, Jul $8 ; 351(2): 125-35$.

[18] D'Amico AV, Chen MH, de Castro M, Loffredo M, Lamb DS, Steigler A, et al. Surrogate endpoints for prostate cancer-specific mortality after radiotherapy and androgen suppression therapy in men with localised or locally advanced prostate cancer: An analysis of two randomised trials. Lancet Oncol 2012, Feb; 13(2): 189-95.

[19] Miyamoto S. A chronological study of hepatic metastasis from colorectal cancer. Jpn J Gastroenterol Sur 1991;24:1990-6.

[20] Kato RB, Srougi V, Salvadori FA, Ayres PP, Leite KM, Srougi M. Pretreatment tumor volume estimation based on total serum psa in patients with localized prostate cancer. Clinics (Sao Paulo) 2008, Dec; 63(6): 759-62.

[21] Babaian RJ, Troncoso P, Steelhammer LC, Lloreta-Trull J, Ramirez EI. Tumor volume and prostate specific antigen: Implications for early detection and defining a window of curability. J Urol 1995, Nov; 154(5): 1808-12.

[22] Tanaka N, Fujimoto K, Hirayama A, Nakai Y, Chihara Y, Anai S, et al. Calculated tumor volume is an independent predictor of biochemical recurrence in patients who underwent retropubic radical prostatectomy. Adv Urol 2012; 2012:204215. 
[23] Vollmer RT, Humphrey PA. Tumor volume in prostate cancer and serum prostatespecific antigen. Analysis from a kinetic viewpoint. Am J Clin Pathol 2003, Jan; 119(1): 80-9.

[24] Carter HB, Pearson JD, Waclawiw Z, Metter EJ, Chan DW, Guess HA, Walsh PC. Prostate-specific antigen variability in men without prostate cancer: Effect of sampling interval on prostate-specific antigen velocity. Urology 1995, Apr; 45(4): 591-6.

[25] Van den Bergh RC, Roemeling S, Roobol MJ, Wolters T, Schröder FH, Bangma CH. Prostate-specific antigen kinetics in clinical decision-making during active surveillance for early prostate cancer--a review. Eur Urol 2008, Sep; 54(3): 505-16.

[26] Ramírez ML, Nelson EC, Devere White RW, Lara PN, Evans CP. Current applications for prostate-specific antigen doubling time. Eur Urol 2008, Aug; 54(2): 291-300.

[27] Lonergan PE, Tindall DJ. Androgen receptor signaling in prostate cancer development and progression. J Carcinog 2011; 10:20.

[28] Bidart JM, Thuillier F, Augereau C, Chalas J, Daver A, Jacob N, et al. Kinetics of serum tumor marker concentrations and usefulness in clinical monitoring. Clin Chem 1999, Oct; 45(10): 1695-707.

[29] Nash AF, Melezinek I. The role of prostate specific antigen measurement in the detection and management of prostate cancer. Endocr Relat Cancer 2000, Mar; 7(1): 37-51.

[30] Ryan CJ, Smith A, Lal P, Satagopan J, Reuter V, Scardino P, et al. Persistent prostatespecific antigen expression after neoadjuvant androgen depletion: An early predictor of relapse or incomplete androgen suppression. Urology 2006;68(4):834-9.

[31] Kelloff GJ, Coffey DS, Chabner BA, Dicker AP, Guyton KZ, Nisen PD, et al. Prostatespecific antigen doubling time as a surrogate marker for evaluation of oncologic drugs to treat prostate cancer. Clin Cancer Res 2004, Jun 1; 10(11): 3927-33.

[32] Oudard S, Banu E, Scotte F, Banu A, Medioni J, Beuzeboc P, et al. Prostate-specific antigen doubling time before onset of chemotherapy as a predictor of survival for hormone-refractory prostate cancer patients. Ann Oncol 2007, Nov; 18(11): 1828-33.

[33] Newling DW. Issues with the use of prostate-specific antigen as a surrogate end point in hormone-resistant prostate cancer. European Urology Supplements 2009, Jan;8(1): 13-9.

[34] Singh AK, Guion P, Susil RC, Citrin DE, Ning H, Miller RW, et al. Early observed transient prostate-specific antigen elevations on a pilot study of external beam radiation therapy and fractionated MRI guided high dose rate brachytherapy boost. Radiat Oncol 2006; 1:28.

[35] Bubley GJ, Carducci M, Dahut W, Dawson N, Daliani D, Eisenberger M, et al. Eligibility and response guidelines for phase II clinical trials in androgen-independent 
prostate cancer: Recommendations from the prostate-specific antigen working group. J Clin Oncol 1999, Nov; 17(11): 3461-7.

[36] WOO TCS, Richard Choo MD, Mary Jamieson RN, Chander BSNDS. Vitamin D3 (cholecalciferol) in the treatment of biochemically-relapsed prostate cancer. .

[37] Guess BW, Scholz MC, Strum SB, Lam RY, Johnson HJ, Jennrich RI. Modified citrus pectin (MCP) increases the prostate-specific antigen doubling time in men with prostate cancer: A phase II pilot study. Prostate Cancer Prostatic Dis 2003;6(4):301-4.

[38] Guess B, Jennrich R, Johnson H, Redheffer R, Scholz M. Using splines to detect changes in PSA doubling times. Prostate 2003, Feb 1; 54(2): 88-94.

[39] Therasse P, Arbuck SG, Eisenhauer EA, Wanders J, Kaplan RS, Rubinstein L, et al. New guidelines to evaluate the response to treatment in solid tumors. European organization for research and treatment of cancer, national cancer institute of the United States, national cancer institute of canada. J Natl Cancer Inst 2000, Feb 2; 92(3): 205-16.

[40] Therasse, P. Response assessment in cancer clinical trials. Doctorate thesis; Erasmus University, Rotterdam, 2006.

[41] Freedland SJ, Humphreys EB, Mangold LA, Eisenberger M, Dorey FJ, Walsh PC, Partin AW. Risk of prostate cancer-specific mortality following biochemical recurrence after radical prostatectomy. JAMA 2005, Jul 27; 294(4): 433-9.

[42] Armstrong AJ, Eisenberger MA, Halabi S, Oudard S, Nanus DM, Petrylak DP, et al. Biomarkers in the management and treatment of men with metastatic castration-resistant prostate cancer. Eur Urol 2012, Mar; 61(3): 549-59.

[43] Gupta S, Carballido E, Fishman M. Sipuleucel-T for therapy of asymptomatic or minimally symptomatic, castrate-refractory prostate cancer: An update and perspective among other treatments. Onco Targets Ther 2011; 4:79-96.

[44] Garcia JA. Sipuleucel-T in patients with metastatic castration-resistant prostate cancer: An insight for oncologists. Ther Adv Med Oncol 2011, Mar; 3(2): 101-8.

[45] Bitting RL, Armstrong AJ, George DJ. Management options in advanced prostate cancer: What is the role for sipuleucel-t? Clinical Medicine Insights: Oncology; 5:325-32.

[46] Stein WD, Gulley JL, Schlom J, Madan RA, Dahut W, Figg WD, et al. Tumor regression and growth rates determined in five intramural NCI prostate cancer trials: The growth rate constant as an indicator of therapeutic efficacy. Clin Cancer Res 2011, Feb 15; 17(4): 907-17.

[47] Madan RA, Bilusic M, Heery C, Schlom J, Gulley JL. Clinical evaluation of TRICOM vector therapeutic cancer vaccines. Semin Oncol 2012, Jun; 39(3): 296-304. 
[48] Kelly WK, Scher HI, Mazumdar M, Vlamis V, Schwartz M, Fossa SD. Prostate-specific antigen as a measure of disease outcome in metastatic hormone-refractory prostate cancer. J Clin Oncol 1993, Apr; 11(4): 607-15.

[49] Smith DC, Dunn RL, Strawderman MS, Pienta KJ. Change in serum prostate-specific antigen as a marker of response to cytotoxic therapy for hormone-refractory prostate cancer. Journal of Clinical Oncology 1998; 16(5): 1835-43.

[50] Berthold DR, Pond GR, Soban F, de Wit R, Eisenberger M, Tannock IF. Docetaxel plus prednisone or mitoxantrone plus prednisone for advanced prostate cancer: Updated survival in the TAX 327 study. J Clin Oncol 2008, Jan 10; 26(2): 242-5.

[51] Berthold DR, Pond GR, de Wit R, Eisenberger M, Tannock IF, TAX 327 Investigators. Survival and PSA response of patients in the TAX 327 study who crossed over to receive docetaxel after mitoxantrone or vice versa. Ann Oncol 2008, Oct; 19(10): 1749-53.

[52] Heidenreich A, Bolla M, Joniau S, Van Der Kwast TH, Matveev V, Mason MD, et al. Guidelines on prostate cancer. Eur Urol 2008; 53(1): 68-80.

[53] Bellmunt J, Rosenberg JE, Choueiri TK. Recent progress and pitfalls in testing novel agents in castration-resistant prostate cancer. Eur Urol 2009; 56(4): 606.

[54] Loriot Y, Massard C, Fizazi K. Recent developments in treatments targeting castration-resistant prostate cancer bone metastases. Ann Oncol 2012, May; 23(5): 1085-94.

[55] Dahut WL, Scripture C, Posadas E, Jain L, Gulley JL, Arlen PM, et al. A phase II clinical trial of sorafenib in androgen-independent prostate cancer. Clin Cancer Res 2008, Jan 1; 14(1): 209-14.

[56] Thuret R, Massard C, Gross-Goupil M, Escudier B, Di Palma M, Bossi A, et al. The postchemotherapy PSA surge syndrome. Ann Oncol 2008, Jul; 19(7): 1308-11.

[57] Chi KN, Ellard SL, Hotte SJ, Czaykowski P, Moore M, Ruether JD, et al. A phase II study of sorafenib in patients with chemo-naive castration-resistant prostate cancer. Ann Oncol 2008, Apr; 19(4): 746-51.

[58] Bellmunt J, Rosenberg JE, Choueiri TK. Recent progress and pitfalls in testing novel agents in castration-resistant prostate cancer. Eur Urol 2009; 56(4): 606.

[59] Bellmunt J, Oh WK. Castration-resistant prostate cancer: New science and therapeutic prospects. Ther Adv Med Oncol 2010, May; 2(3): 189-207.

[60] Rixe O, Fojo T. Is cell death a critical end point for anticancer therapies or is cytostasis sufficient? Clin Cancer Res 2007, Dec 15; 13(24): 7280-7.

[61] Nabhan C, Tolzien K, Lestingi T, Kelby SK, Galvez AG, Bitran JD. Activity of sorafenib (SOR) in chemotherapy-failure castration-resistant prostate cancer (CRPC). ASCO/GU Proceedings 2010; 122. 
[62] Carmichael C, Lau C, Josephson DY, Pal SK. Comprehensive overview of axitinib development in solid malignancies: Focus on metastatic renal cell carcinoma. Clin Adv Hematol Oncol 2012 May; 10(5): 307-14.

[63] Baselga J, Campone M, Piccart M, Burris HA, Rugo HS, Sahmoud T, et al. Everolimus in postmenopausal hormone-receptor-positive advanced breast cancer. N Engl J Med 2012, Feb 9; 366(6): 520-9.

[64] Kung HJ. Targeting tyrosine kinases and autophagy in prostate cancer. Horm Cancer 2011, Feb; 2(1): 38-46.

[65] Adamo V, Noto L, Franchina T, Chiofalo G, Picciotto M, Toscano G, Caristi N. Emerging targeted therapies for castration-resistant prostate cancer. Front Endocrinol (Lausanne) 2012; 3:73.

[66] Agarwal N, Sonpavde G, Sternberg CN. Novel molecular targets for the therapy of castration-resistant prostate cancer. Eur Urol 2012, May; 61(5): 950-60.

[67] Brindle K. Watching tumours gasp and die with MRI: The promise of hyperpolarised 13C MR spectroscopic imaging. Br J Radiol 2012, Jun; 85(1014): 697-708.

[68] Rosen MA. Use of modified RECIST criteria to improve response assessment in targeted therapies: Challenges and opportunities. Cancer Biol Ther 2010, Jan; 9(1): 20-2.

[69] Mehrara E, Forssell-Aronsson E, Ahlman H, Bernhardt P. Specific growth rate versus doubling time for quantitative characterization of tumor growth rate. Cancer Res 2007, Apr 15; 67(8): 3970-5.

[70] Mehrara E, Forssell-Aronsson E, Ahlman H, Bernhardt P. Quantitative analysis of tumor growth rate and changes in tumor marker level: Specific growth rate versus doubling time. Acta Oncol 2009; 48(4): 591-7.

[71] Mehrara E, Forssell-Aronsson E, Bernhardt P. Objective assessment of tumour response to therapy based on tumour growth kinetics. Br J Cancer 2011, Aug 23; 105(5): 682-6.

[72] Elmore S. Apoptosis: A review of programmed cell death. Toxicol Pathol 2007, Jun; 35(4): 495-516.

[73] Eisenhauer EA, Therasse P, Bogaerts J, Schwartz LH, Sargent D, Ford R, et al. New response evaluation criteria in solid tumours: Revised RECIST guideline (version 1.1). Eur J Cancer 2009, Jan; 45(2): 228-47.

[74] Weber WA. Assessing tumor response to therapy. J Nucl Med 2009, May; 50 Suppl 1:1S-10S.

[75] Le Tourneau C, Servois V, Diéras V, Ollivier L, Tresca P, Paoletti X. Tumour growth kinetics assessment: Added value to RECIST in cancer patients treated with molecularly targeted agents. Br J Cancer 2012, Feb 28; 106(5): 854-7.

[76] Ferte C, Albiges L, Soria JC, Loriot Y, Fizazi K, Escudier BJ. The use of tumor growth rate (TGR) in evaluating sorafenib and everolimus treatment in mrcc patients: An in- 
tegrated analysis of the TARGET and RECORD phase III trials data. J Clin Oncol 30, 2012 (suppl; Abstr 4540).

[77] Motulsky H. Intuitive statistics a nonmathematical guide to statistical thinking. Second Ed. New York: Oxford University Press; 2010k.

[78] Nishida K, Kaneko T, Yoneda M, Nakagawa S, Ishikawa T, Yamane E, et al. Doubling time of serum CA 19-9 in the clinical course of patients with pancreatic cancer and its significant association with prognosis. J Surg Oncol 1999, Jul; 71(3): 140-6.

[79] Smith MR, Manola J, Kaufman DS, Oh WK, Bubley GJ, Kantoff PW. Celecoxib versus placebo for men with prostate cancer and a rising serum prostate-specific antigen after radical prostatectomy and/or radiation therapy. J Clin Oncol 2006, Jun 20; 24(18): 2723-8.

[80] Srinivas S, Feldman D. A phase II trial of calcitriol and naproxen in recurrent prostate cancer. Anticancer Res 2009, Sep; 29(9) : 3605-10.

[81] Hanninen M, Venner P, North S. A rapid PSA half-life following docetaxel chemotherapy is associated with improved survival in hormone refractory prostate cancer. Can Urol Assoc J 2009, Oct; 3(5): 369-74.

[82] De Crevoisier R, Slimane K, Messai T, Wibault P, Eschwege F, Bossi A, et al. Early PSA decrease is an independent predictive factor of clinical failure and specific survival in patients with localized prostate cancer treated by radiotherapy with or without androgen deprivation therapy. Ann Oncol 2010, Apr; 21(4): 808-14.

[83] Lee JH, Gulec SA, Kyshtoobayeva A, Sim MS, Morton DL. Biological factors, tumor growth kinetics, and survival after metastasectomy for pulmonary melanoma. Ann Surg Oncol 2009, Oct; 16(10): 2834-9. 